\title{
Computing Hereditary Convex Structures
}

\author{
Bernard Chazelle $\cdot$ Wolfgang Mulzer
}

Received: 30 June 2009 / Revised: 19 March 2010 / Accepted: 12 May 2010 /

Published online: 25 March 2011

(C) Springer Science+Business Media, LLC 2011

\begin{abstract}
Color red and blue the $n$ vertices of a convex polytope $\mathcal{P}$ in $\mathbb{R}^{3}$. Can we compute the convex hull of each color class in $o(n \log n)$ time? What if we have more than two colors? What if the colors are random? Consider an arbitrary query halfspace and call the vertices of $\mathcal{P}$ inside it blue: can the convex hull of the blue points be computed in time linear in their number? More generally, can we quickly compute the blue hull without looking at the whole polytope? This paper considers several instances of hereditary computation and provides new results for them. In particular, we resolve an eight-year old open problem by showing how to split a convex polytope in linear expected time.
\end{abstract}

Keywords Convex polytope $\cdot$ Halfspace range searching $\cdot$ Hereditary convex hulls

\section{Introduction}

Given a set of $n$ points in the Euclidean plane and its Voronoi diagram, it was shown in [13] how to compute the Voronoi diagram of any given subset in linear time. ${ }^{1}$ The authors asked whether it is also possible to compute the convex hull of an arbitrary

\footnotetext{
${ }^{1}$ All our algorithms are randomized, so the complexity is to be understood in the expected sense. Note that the expectation is only over the randomness used by the algorithm and that the complexity bounds hold for every input, except when explicitly stated otherwise.
}

This work was supported in part by NSF grant CCF-0634958 and NSF CCF 0832797. W. Mulzer was supported by a Wallace Memorial Fellowship in Engineering.

B. Chazelle

Department of Computer Science, Princeton University, 35 Olden Street, Princeton, NJ 08540, USA

e-mail: chazelle@cs.princeton.edu

W. Mulzer (殴

Institut für Informatik, Freie Universität Berlin, Takustraße 9, 14195 Berlin, Germany

e-mail: mulzer@inf.fuberlin.de 
subset of the vertices of a convex 3-polytope in linear time. An affirmative answer would, of course, imply the previous result. This paper proves that it is indeed the case. We formulate the question in a hereditary setting by assuming that the vertices of a convex polytope $\mathcal{P}$ in $\mathbb{R}^{3}$ are colored red and blue. The problem is then to "split" $\mathcal{P}$ and compute both monochromatic convex hulls; see Fig. 1. We show how to do this in linear time, which answers the main open question in [13]. We extend our techniques to an arbitrary number of colors by showing how to compute the convex hulls of all the color classes in $O(n \sqrt{\log n})$ time. In subsequent work, Kevin Buchin and the second author improved this bound to $O\left(n(\log \log n)^{2}\right)$ time [6]. Interestingly, we can do this in linear time for any set of $\chi$ colors, as long as the coloring is random; the result holds for any $1 \leq \chi \leq n$. We also consider the coloring induced by halfspace range queries: given a query plane, compute the convex hull of the points lying on one side. We show how to do so in time $O(\log n+k)$, where $k$ is the output size; the data structure requires $O(n \log n)$ storage.

Our offline splitting algorithm cannot be output-sensitive, since the output size is linear. But what if we output only one color class? If the chosen vertices form $k$ connected components in the skeleton graph of $\mathcal{P}$, we can compute their convex hull in time $O\left(n \log ^{*} n+k \log k\right)$, where $n$ now is the size of the subset. Our result has this intriguing corollary: given a Delaunay triangulation (DT) denoted by $\mathcal{T}$, the DT of any set $S$ of $n$ vertices and edges in $\mathcal{T}$ can be computed in time $O\left(n \log ^{*} n+k \log k\right)$, where $k$ is the number of connected components formed by $S$ within $\mathcal{T}$. We actually prove a slightly more general result. It is well known that the convex hull of two convex polytopes can be stitched together in linear time [11]. We consider the case of $k$ disjoint convex polytopes with a total of $n$ vertices. If the vertices of each polytope form a connected component in the convex hull of their union, we can compute their common convex hull in $O(n \log * n+k \log k)$ time. This assumption is motivated by a lower bound of $\Omega(n \log k)$ for the general case.

To study the complexity of hereditary computing is part of a broader attempt to understand what makes what hard. To compute the DT of $n$ points in the plane requires $\Omega(n \log n)$ time, but knowing that the points are the vertices of a convex polygon cuts down the complexity to linear $[1,14]$. Given a spanning subgraph of degree at most $d$, the DT can be completed in time $O\left(n d \log ^{*} n\right)$ [21]. In fact, at the cost of a more complicated algorithm, it can be done in linear time [17, 27]. Furthermore, Djidjev and Lingas have proven linearity for any set of points forming a monotone chain in both $x$ and $y$ directions [22]. This might suggest that the hardness of DT is really confined to sorting, but the situation is more complicated. Sorting helps to find Voronoi diagrams in $\ell_{\infty}$ [15], but ranking the points in any one direction still leaves us with a $\Theta(n \log n)$ complexity [22]. If we know the order in $x$ and $y$ direction, the DT can be found with $O(n)$ comparisons, but no $o(n \log n)$ algorithm is known [6]. More generally, the simplicity of a polygon is known to "linearize" many problems that otherwise exhibit $\Omega(n \log n)$ lower bounds, e.g., polygon triangulation [2, 10, $33]$, medial axis [16], or constrained Delaunay triangulation [17, 27]. Our work fits into that mold.

Hereditary algorithms are nothing new. Given a subset of a simple polygon, Chan [8] showed how to compute its convex hull in linear time ${ }^{2}$ and how to trian-

\footnotetext{
${ }^{2}$ Here, linear time means linear in the size of the whole structure, not just the subset.
} 

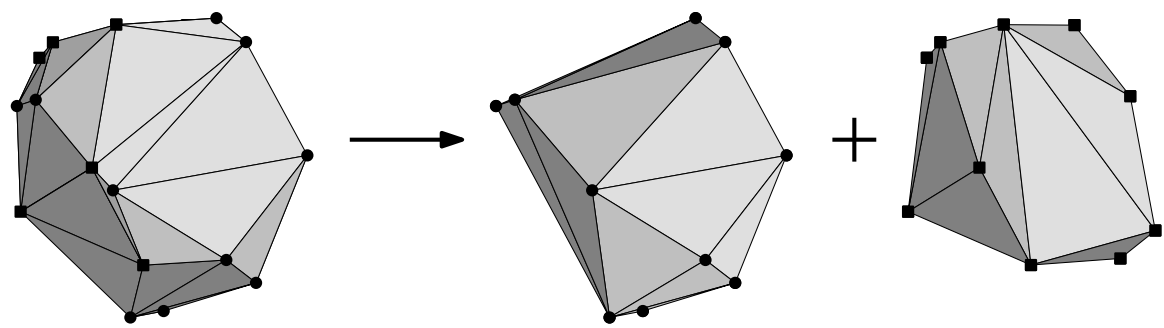

Fig. 1 Given their joint convex hull, we can find the red and blue hulls in linear time

Fig. 2 General hereditary trapezoidal decompositions are hard

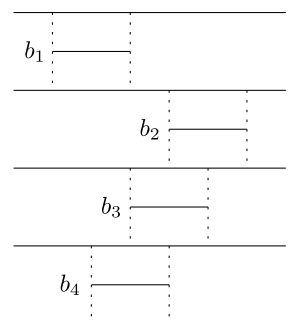

gulate it in $O\left(n \log ^{*} n\right)$ time. Van Kreveld, Löffler, and Mitchell [28] improved the latter result by proving that any subset of a given triangulation can in fact be triangulated in linear time. To appreciate the difficulty of obtaining general hereditary algorithms, let us mention the example of hereditary trapezoidal decompositions [8, 26]. Kirkpatrick, Klawe, and Tarjan [26] gave an algorithm for removing a hole-free subset of line segments in a trapezoidal decomposition in linear time, where holefreeness is a property that is necessary to ensure that the subset does not obscure too much information. They also give an example that for general hereditary trapezoidal decompositions, no improvement is possible (see also [8]). Consider the line segments in Fig. 2 and their trapezoidal decomposition. Suppose that we would like to find the trapezoidal decomposition of $b_{1}, b_{2}, b_{3}, b_{4}$. To achieve this, we essentially have no choice but to sort their endpoints from scratch, since the long line segments obscure all information. This means that trapezoidal decompositions, unlike convex hulls, do not always give away something about their subsets. There are many other situations in which additional "hereditary" information brings no benefits: if $P$ is a point set in $\mathbb{R}^{3}$, sorting $P$ in a bounded number of directions does not help in computing its convex hull [32]; nor does knowing the convex hull of $P$ help in finding its diameter [25].

Another way to look at our first result, the linear complexity of bicolored convex hulls, is that the convex hull problem in $3 \mathrm{D}$ loses its $\Omega(n \log n)$-hardness if it is embedded in a larger polytope: in other words, computationally speaking, a convex polytope "gives away" the convex hull of any of its subsets. 


\section{Definitions and Notation}

Given a finite point set $P \subseteq \mathbb{R}^{3}$, let conv $P$ denote the convex hull of $P$. We denote the edges and facets of conv $P$ by $E[P]$ and $F[P]$. For a point $p \in P$, let $\operatorname{deg}_{P} p$ be the number of edges in $E[P]$ incident to $p$, the degree of $p$ (with respect to $P$ ). Throughout, we will assume that convex hulls are given in a standard planar graph representation, e.g., a DCEL [4, Chap. 2.2]. Our point sets will usually be in general convex position (gcp), i.e., every three points in $P$ are linearly independent, and $p \notin$ $\operatorname{conv}(P \backslash p)$ for every $p \in P$. In particular, conv $P$ is simplicial, ${ }^{3}$ and all the points in $P$ are vertices of conv $P$.

We use classical geometric random sampling [19, 30]; see Appendix A. For this, we quickly review the notion of conflict sets. Given a point set $P \subseteq \mathbb{R}^{3}$, an edge $e \in E[P]$, and a point $p \notin \operatorname{conv} P$, we say that $p$ can see $e$ in conv $P$ or that $e$ is visible from $p$ if the triangle spanned by $e$ and $p$ intersects conv $P$ only in $e$. All the planes we consider are oriented, that is, one of the two halfspaces defined by a plane $h$ is designated the left halfspace of $h, h^{+}$, and the other one is designated the right halfspace of $h, h^{-}$. We use the convention that every supporting plane of conv $P$ is oriented such that $P$ lies in the right halfspace $h^{-}$. Let $Q \subseteq P, f \in F[Q]$, and $h_{f}$ be the supporting plane for $f$. A point $p \in P$ is in conflict with $f$ if $p$ lies in $h_{f}^{+}$. Let $B_{f} \subseteq P$ denote the points in conflict with $f$, and $b_{f}$ the size of $B_{f}$. Conversely, for a point $p \in P$, we let $D_{p} \subseteq F[Q]$ denote the set of facets in conflict with $p$, and let $d_{p}$ be its size. The sets $B_{f}$ and $D_{p}$ are the conflict sets of $f$ and $p$, and $b_{f}$ and $d_{p}$ are the conflict sizes. By double counting,

$$
\sum_{f \in F[Q]} b_{f}=\sum_{p \in P} d_{p} .
$$

\section{Splitting Polytopes}

We are given an $n$-point set $P \subseteq \mathbb{R}^{3}$ in general convex position (gcp). Let $B \subseteq P$, and let $R=P \backslash B$. The points in $B$ are called blue, the points in $R$ are called red. Given conv $P$, we show how to obtain conv $B$ in linear time.

Theorem 3.1 Let $P \subseteq \mathbb{R}^{3}$ be a set of $n$ points in general convex position, colored red and blue. Given conv $P$, the convex hull of the blue points can be computed in $O(n)$ expected time.

An edge of conv $P$ is called blue if both of its endpoints are blue, and red if both of its endpoints are red, otherwise it is bichromatic. Blue, red, and bichromatic facets are defined similarly. The splitting is performed by a recursive algorithm SplitHull that receives the convex hull and a two-coloring of $P$. Please refer to Algorithm 1.

\footnotetext{
${ }^{3}$ That is, all facets of conv $P$ are triangles.

${ }^{4}$ See Sect. 2 for basic definitions and notation.
} 
Algorithm 1 Splitting a bichromatic convex hull

SplitHull(conv $P$ )

1. If $P$ contains no red points, return conv $P$.

2. If there exists a red point $r$ in $P$ with $\operatorname{deg}_{P} r \leq d_{0}$ (with a suitable constant $d_{0}$ ), then return SplitHull( $\operatorname{conv}(P \backslash r))$.

3. Take random blue points $b \in B$ until (i) $\operatorname{deg}_{P} b \leq 6$, and (ii) there exists a blue edge $e$ in conv $(P \backslash b)$ that is visible from $b$.

4. Call SplitHull (conv $(P \backslash b))$ to compute conv $(B \backslash b)$.

5. Using $e$ as a starting edge, insert $b$ into conv $(B \backslash b)$ and return conv $B$.

SplitHull can be seen as a generalization of Chew's algorithm for Voronoi diagrams of convex polygons [14], and it is also reminiscent of Dobkin and Kirkpatrick's hierarchy [23, 24]. It first tries to delete a red point of small degree. If this is not possible, it removes blue points until there is a red point of small degree again. Later, these blue points must be reinserted into the recursively computed blue hull. In order to do this efficiently, we must be careful about which blue points we delete, so that we have a landmark from where to start the conflict location. SplitHull is easily shown to be correct.

Lemma 3.2 SplitHull(conv $P$ ) computes conv $B$.

Proof The proof is by straightforward induction on $|P|$. We only comment on Step 5 . Let $B^{-}=B \backslash b$ and $P^{-}=P \backslash b$. If $e$ is a blue edge visible from $b$ in conv $P^{-}$, then the same holds in conv $B^{-}$: since $e$ has both endpoints in $B^{-}$, a supporting plane for $e$ in conv $P^{-}$supports $e$ also in conv $B^{-}$, and since conv $B^{-} \subseteq$ conv $P^{-}$, the triangle spanned by $b$ and $e$ intersects conv $B^{-}$only in $e$. Thus, we can walk from $e$ to determine $b$ 's conflict set $D_{b}$ and replace $D_{b}$ by new facets incident to $b$. This takes time $O\left(\left|D_{b}\right|\right)$ [4, Chap. 11.2]. When implementing the algorithm, care must be taken that the pointer to $e$ obtained in Step 3 is not invalidated by the recursive call in Step 4. We can easily do it as follows: when deleting a blue edge in Step 4, retain the corresponding record in memory and reuse it when the edge is recreated in Step 5.

The bulk of the analysis lies in bounding the running time.

Lemma 3.3 The expected time needed for one invocation of SplitHull is constant, not counting the time for the recursive calls.

Proof We argue that each step takes constant expected time. This clearly holds for Step 1: just use a counter for the number of red points. Step 2 is also easy: keep a linked list $L$ for the red points with degree at most $d_{0}$. During preprocessing, determine the degrees and initialize $L$ accordingly. When the hull is altered in Steps 2 and 4 , update the degrees and $L$. Since all relevant vertices have bounded degree, this takes constant time. The most interesting part lies in the analysis of Step 3. We show that there is a good chance of sampling a point with the required properties. 
Lemma 3.4 Let $\widetilde{B}$ be the subset of the blue points $b$ with the following properties: (i) $\operatorname{deg}_{P} b \leq 6$; and (ii) $b$ is a vertex of a blue facet of conv $P$, or $E[P \backslash b] \backslash E[P]$ contains at least one blue edge. ${ }^{5}$ There exists a constant $d_{0}$ such that if all red points have degree at least $d_{0}$, then $|\widetilde{B}| \geq|P| / 5$.

Proof Call a blue point pleasant if it satisfies the properties in the lemma, and ghastly otherwise. By Euler's formula, a large fraction of blue points has degree at most 6 . If a blue point $b$ is ghastly and has degree at most 6 , then either (a) $b$ is incident to a facet with a red edge, or (b) $b$ 's neighborhood has only bichromatic edges and to delete $b$ from conv $P$ creates no blue edge. We bound the number of points satisfying (a) and (b) separately and then finish the analysis with a union bound.

In the following, we will assume that $d_{0}$ is a large enough constant. By general convex position, we have $|E[P]|=3 n-6 .{ }^{6}$ Let $B^{\prime}$ be the set of blue points $b$ with $\operatorname{deg}_{P} b \leq 6$. Since conv $P$ is three-connected [29, Theorem 5.3.3], and since all red nodes have degree at least $d_{0} \geq 7$, we get

$$
6 n-12=\sum_{p \in B^{\prime}} \operatorname{deg} p+\sum_{p \in P \backslash B^{\prime}} \operatorname{deg} p \geq 3\left|B^{\prime}\right|+7\left(n-\left|B^{\prime}\right|\right) .
$$

Thus,

$$
\left|B^{\prime}\right|>n / 4 \text {. }
$$

Similarly,

$$
6 n-12=\sum_{p \in R} \operatorname{deg} p+\sum_{p \in P \backslash R} \operatorname{deg} p \geq d_{0}|R|+3(n-|R|)=\left(d_{0}-3\right)|R|+3 n,
$$

so $|R|<4 n / d_{0}$ (for $d_{0} \geq 12$ ). Let $E_{R}$ denote the set of red edges in conv $P$. Since every red edge of conv $P$ is an edge of conv $R$,

$$
\left|E_{R}\right| \leq|E[R]|=3|R|-6<12 n / d_{0} .
$$

For $b \in B^{\prime}$, let $\Gamma_{b}$ be the simple polygon formed by $b$ 's neighbors in conv $P$, and let $C$ be the set of points $b \in B^{\prime}$ such that $\Gamma_{b}$ contains a red edge (this corresponds to property (a) mentioned at the beginning of the proof). Since an edge is incident to two facets, for each $e \in E_{R}$, there are at most two points $p, q \in C$ such that $e$ is in $\Gamma_{p}$ and $\Gamma_{q}$. Hence, by (3),

$$
|C| \leq 2\left|E_{R}\right|<24 n / d_{0} .
$$

Now, let $D \subseteq B^{\prime}$ be the set of points $b$ such that $\Gamma_{b}$ has no monochromatic edge. For any such $b, \operatorname{deg}_{P} b$ is even, and red and blue points alternate along $\Gamma_{b}$. Let $E_{b}=$ $E[P \backslash b] \backslash E[P]$. We say that $b$ creates $E_{b}$. Note that $E_{b}$ contains only diagonals of $\Gamma_{b}$. Any edge $e$ is created by at most two points in $D$ : if $e$ is occluded in conv $P$ by

\footnotetext{
${ }^{5}$ Recall that $E[P], F[P]$ denote the edges and facets of conv $P$ (see Sect. 2).

${ }^{6}$ Since all the points are on the hull, Euler's formula $[5$, Theorem 7.2.1] yields $n-|E[P]|+|F[P]|=2$, and since all facets are triangles, we have $2 \mid[E[P]|=3| F[P] \mid$.
} 
Fig. 3 (a) The edge $e_{1}$ is occluded by exactly one edge and is created by $u$ and $v$; (b) the edge $e_{2}$ is occluded by two edges and is created only by $w$

Fig. 4 Every triangulation of a two-colored simple polygon contains at least one monochromatic diagonal (shown in dashed)

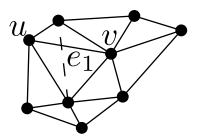

(a)

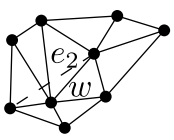

(b)

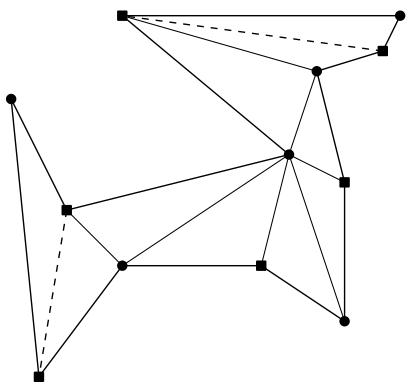

exactly one edge, it is created by the endpoints of this edge; if $e$ is occluded by two or more edges, it can only be created by a point incident to all of them; see Fig. 3. Furthermore, every $b \in D$ creates at least one monochromatic edge, since every triangulation of a two-colored simple polygon contains at least one monochromatic diagonal; ${ }^{7}$ see Fig. 4 . Let $D^{\prime}$ be the set of points in $D$ that do not create a blue edge (these are the points with property (b)). By the previous discussion and (3),

$$
\left|D^{\prime}\right| \leq 2|E[R]|<24 n / d_{0} .
$$

To conclude, we observe that all the points in the set $B^{\prime} \backslash\left(C \cup D^{\prime}\right)$ are pleasant and that by $(2,4,5)$ it contains at least $\left(1 / 4-48 / d_{0}\right) n>n / 5$ points, for $d_{0}$ large enough.

By Lemma 3.4 we expect at most five iterations in Step 3, each taking constant time, since all points under consideration have bounded degree. The same holds for Step 4 without the recursive call, as $\operatorname{deg}_{P} b \leq 6$. Finally, we use backwards analysis to handle Step 5. Take $\widetilde{B}$ as in Lemma 3.4. Because $|\widetilde{B}|>|B| / 5$, the average degree of a point in $\widetilde{B}$ is less than 30 , by Euler's formula. Hence, to delete a random point $b \in \widetilde{B}$ from conv $B$ takes constant expected time, and this is exactly the cost of inserting $b$ into conv $(B \backslash b)$ [4, Chap. 11.2].

Theorem 3.1 follows from Lemmas 3.2 and 3.3, since the number of recursive calls is $O(n)$.

\footnotetext{
${ }^{7}$ Since the dual graph of this triangulation is a tree [4, Sect. 3.1], and every tree contains at least one leaf, corresponding to a triangle between two adjacent edges.
} 
$(\alpha)$

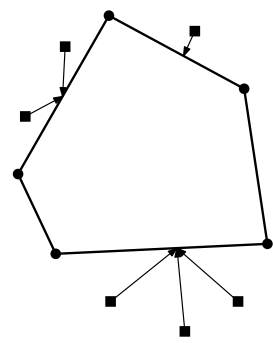

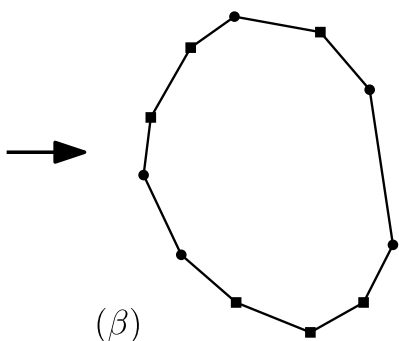

$(\beta)$

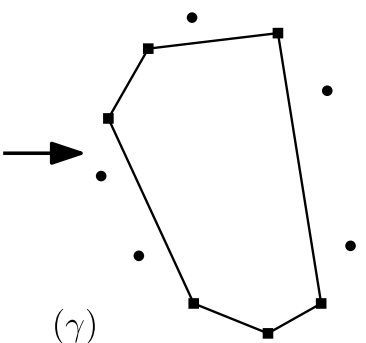

Fig. 5 Splitting random colorings: the algorithm $(\alpha)$ computes conv $S$ and conflict facets for $C_{i},(\beta)$ inserts $C_{i}$ into conv $S$, and $(\gamma)$ extracts conv $C_{i}$. The points in $C_{i}$ are shown as boxes, $S$ as circles

\begin{tabular}{l} 
Algorithm 2 Splitting random colorings \\
\hline RandMultiSplit (conv $P$ ) (* see Fig. $\left.5^{*}\right)$
\end{tabular}

1. Pick a random sample $S \subseteq P$ of size $n / \chi$ and compute conv $S$.

2. For each $p \in P$, determine a facet $f_{p} \in F[S]$ in conflict with $p$.

3. For each color $i$ :

(a) Insert all points of $C_{i}$ into conv $S$.

(b) Extract conv $C_{i}$ from conv $\left(C_{i} \cup S\right)$.

\section{Splitting Random Colorings}

Now, we extend SplitHull to handle more than two colors: for a point set $P \subseteq \mathbb{R}^{3}$, let $c: P \rightarrow\{1, \ldots, \chi\}$ be a coloring of $P$. For $i \in\{1, \ldots, \chi\}$, we let $C_{i}=c^{-1}(i)$ denote the points that are colored $i$, the $i$ th color class. The coloring $c$ is called random if each point $p$ is colored uniformly and independently with a color in $\{1, \ldots, \chi\}$. This section deals with random colorings; and the next one is about arbitrary colorings.

Theorem 4.1 Let $P \subseteq \mathbb{R}^{3}$ be a set of $n$ points in general convex position, and let $c: P \rightarrow\{1, \ldots, \chi\}$ be a random coloring of $P$. Given conv $P$, we can compute the convex hulls conv $C_{1}, \ldots, \operatorname{conv} C_{\chi}$ in $O(n)$ expected time (the expectation is over the coloring and the random choices of the algorithm).

The algorithm for Theorem 4.1 is called RandMultiSplit. See Algorithm 2. It receives the convex hull and a coloring of $P$ as input, and it computes the convex hull of a random sample $S \subseteq P$ into which the points of each color class are then inserted separately. As we will see below, this can be done quickly because $c$ is random. Finally, it uses SplitHull to remove the points from $S$.

Clearly, the algorithm correctly computes the $\operatorname{conv} C_{i}$. We bound the running time of each step. Using SplitHull, Step 1 requires $O(n)$ time. The analysis of Step 2 needs more work.

Lemma 4.2 Step 2 takes $O(n)$ expected time. 
Fig. 6 Claim 4.3: the facets $F[Q]$ are shown dashed, $F[P]$ solid. Merge $\Gamma_{P}(p)$ with $\Gamma_{Q}(p)$ to determine its conflict facets

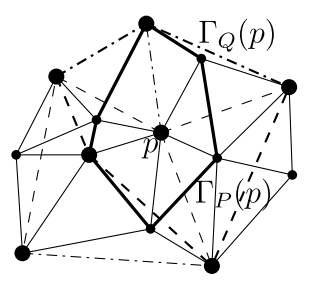

Algorithm 3 Determining the conflict facets in a subset

SubsetConflictWalk(conv $S$, conv $P$ )

1. Let queue be a queue with the elements in $S$.

2. While queue $\neq \varnothing$.

(a) Let $p$ be the next point in queue.

(b) If $p \notin S$, insert $p$ into conv $S$, using a previously computed conflict facet $f_{p}$ for $p$ as a starting point.

(c) For each neighbor $q \in \Gamma_{P}(p)$, find a conflict facet $\tilde{f}_{q}$ in conv $(S \cup p)$, using Claim 4.3.

(d) Using the $\tilde{f}_{q}$ 's, find conflict facets $f_{q} \in F[S]$ for all $q \in \Gamma_{P}(p)$. If $q \in \Gamma_{P}(p)$ has not been encountered yet, insert it into queue.

Proof For $Q \subseteq P$ and $p \in Q$, let $\Gamma_{Q}(p)$ denote the neighbors of $p$ in conv $Q$. First, we show how to compute the conflict facets for points that are neighbors in conv $P$ of a point in $Q$.

Claim 4.3 Let $Q \subseteq P$ and $p \in Q$. Assume that both conv $Q$ and conv $P$ are available. In time $O\left(\operatorname{deg}_{Q} p+\operatorname{deg}_{P} p\right)$, we can compute a conflict facet $f_{q} \in F[Q]$ for every neighbor $q \in \Gamma_{P}(p)$ of $p$.

Proof Consider an overlay of conv $Q$ and conv $P$, i.e., a central projection of their vertices and edges onto the unit sphere centered at a point $O \in \operatorname{conv} Q$. Let $q \in$ $\Gamma_{P}(p)$, and let $f \in F[Q]$ be the facet incident to $p$ that is intersected by the line segment $p q$ in the overlay. Then $q$ is in conflict with $f$. To see this, let $h_{f}$ be the plane supporting $f$. If $q$ did not conflict with $f$, then $q$ would lie in $h_{f}^{-}$, and at least part of the line segment $p q$ would be strictly inside conv $Q$. But then $p q$ could not be an edge of conv $P$, as conv $Q \subseteq$ conv $P$. Thus, conflict facets for $\Gamma_{P}(p)$ can be computed by merging the cyclically ordered lists $\Gamma_{P}(p)$ and $\Gamma_{Q}(p)$ with respect to some overlay of the hulls; see Fig. 6. This takes time $O\left(\operatorname{deg}_{Q} p+\operatorname{deg}_{P} p\right)$.

The conflict facets for $P$ can now be found by breadth-first search, using an algorithm called SubsetConflictWalk. Please refer to Algorithm 3. Step 2 of the algorithm maintains the invariant that a conflict facet $f_{p} \in F[S]$ is known for each $p \in$ queue $\backslash S$. Using standard techniques, Step 2(b) takes $O\left(d_{p}\right)$ time, where $d_{p}$ is 
the conflict size of $p$ in conv $S$ [4, Chap. 11.2]. ${ }^{8}$ Furthermore, by Claim 4.3, the conflict facets of $\Gamma_{P}(p)$ can be found in $O\left(\operatorname{deg}_{S \cup p} p+\operatorname{deg}_{P} p\right)$ time. Finally, Step 2(d) takes time $O\left(\operatorname{deg}_{P} p\right)$ : every facet $\tilde{f} \in F[S \cup p]$ shares at least one edge $e$ with an $f \in F[S]$, and if $q$ can see $e$ in conv $S$, it conflicts with at least one facet adjacent to $e$. Thus, $f_{q}$ can be computed from $\tilde{f}_{q}$ in constant time. It follows that the total running time of SubsetConflictWalk is proportional to

$$
\mathbf{E}\left[\sum_{p \in P}\left(d_{p}+\operatorname{deg}_{S \cup p} p+\operatorname{deg}_{P} p\right)\right] .
$$

Now, since ${ }^{9} \operatorname{deg}_{S \cup p} p \ll d_{p}$ for $p \notin S$, this is proportional to

$$
\mathbf{E}\left[\sum_{p \in S} \operatorname{deg}_{S} p+\sum_{p \in P \backslash S} d_{p}+\sum_{p \in P} \operatorname{deg}_{P} p\right] \ll \mathbf{E}\left[\frac{n}{\chi}+\sum_{f \in F[S]} b_{f}+n\right],
$$

by (1) in Sect. 2. The lemma follows, since $\mathbf{E}\left[\sum_{f \in F[S]} b_{f}\right] \ll n$ by Lemma A.4(8) $\left(b_{f}\right.$ is the conflict size of $f$ ).

Now we consider Step 3 of RandMultiSplit. Fix a color $i$, and for each $f \in$ $F[S]$, let $a_{f}=\left|C_{i} \cap B_{f}\right|$. Since the coloring is random, conditioned on $b_{f}$, the size $a_{f}$ is distributed like a sum of independent Bernoulli random variables with mean $b_{f} / \chi$. By standard moment bounds [12, Lemma A.1], $\mathbf{E}_{c}\left[a_{f}^{2}\right] \ll\left(b_{f} / \chi\right)^{2}$. By Lemma A.5, Step 3(a) takes time $\mathbf{E}_{S, c}\left[\sum_{f \in F[S]} a_{f} \log a_{f}\right]$, and by Lemma A.4(8), we get

$$
\begin{aligned}
\mathbf{E}_{S, c}\left[\sum_{f \in F[S]} a_{f} \log a_{f}\right] & \ll \mathbf{E}_{S}\left[\sum_{f \in F[S]} \mathbf{E}_{c}\left[a_{f}^{2}\right]\right] \ll \mathbf{E}_{S}\left[\frac{1}{\chi^{2}} \sum_{f \in F[S]} b_{f}^{2}\right] \\
& \ll \frac{\chi n}{\chi^{2}}=\frac{n}{\chi} .
\end{aligned}
$$

Using SplitHull in Step 3(b), conv $C_{i}$ can now be computed in time $O\left(\left|C_{i}\right|+\right.$ $n / \chi)$. There are $\chi$ colors, so Step 3 takes total time proportional to $\sum_{i}\left|C_{i}\right|+\chi$. $(n / \chi) \ll n$, and Theorem 4.1 follows.

\section{Splitting Arbitrary Colorings}

We now consider arbitrary colorings. With SplitHull as a black box, we can easily split a $\chi$-colored polytope in time $O(n \log \chi)$. For sufficiently large $\chi$, this can be improved.

Theorem 5.1 Let $P \subseteq \mathbb{R}^{3}$ be a set of $n$ points in general convex position, and let $c: P \rightarrow\{1, \ldots, \chi\}$ be an arbitrary coloring of $P$. Given conv $P$, we can compute conv $C_{1}, \ldots, \operatorname{conv} C_{\chi}$ in $O(n \sqrt{\log n})$ expected time.

\footnotetext{
${ }^{8} d_{p}=0$ if $p \in S$.

${ }^{9}$ We use the Vinogradov notation $f \ll g$ for $f=O(g)$ and $f \gg g$ for $f=\Omega(g)$.
} 
For the random colorings in the previous section, we could exploit the fact that each color is spread uniformly over the polytope in order to design a simple divide and conquer algorithm that decomposes each color class into subsets of expected constant size. This is no longer possible for arbitrary colorings, because now the distribution of color classes can be highly irregular. Therefore, we need a more sophisticated scheme to partition the color classes. We begin with a useful sampling lemma.

Lemma 5.2 Let $Q \subseteq \mathbb{R}^{3}$ be an m-point set in general convex position, and let $\mu \in$ $(0,1)$ be a constant. There exists an integer $\alpha_{0}$ such that the following holds: let $\alpha \in\left\{\alpha_{0}, \ldots, \mu m\right\}$. Given conv $Q$, in $O(m)$ time we can compute subsets $S, R \subseteq Q$ and a partition $R_{1}, \ldots, R_{\beta}$ of $R$ such that

1. $|S|=\alpha,|R| \gg m$, and $\max _{i}\left|R_{i}\right| \ll m(\log \alpha) / \alpha$.

2. For each $R_{i}$, there exists a facet $f_{i} \in F[S]$ such that all points in $R_{i}$ are in conflict with $f_{i}$.

3. Every point in $R$ conflicts with constantly many facets of conv $S$.

4. The conflict sets for two points $p \in R_{i}, q \in R_{j}, i \neq j$, are disjoint and no conflict facet of $p$ shares an edge with a conflict facet of $q$.

Furthermore, the convex hulls conv $S$, conv $R_{1}, \ldots$, conv $R_{\beta}$, conv $(Q \backslash(R \cup S))$ can be computed in expected $O(m)$ time.

Proof We call a subset $S \subseteq Q$ decent if it has two properties: (i) $\sum_{f \in F[S]} b_{f} \ll m$; and (ii) $\max _{f \in F[S]} b_{f} \ll m(\log \alpha) / \alpha$, where $b_{f}$ denotes the conflict size of $f$.

Claim 5.3 A decent subset $S \subseteq Q$ of size $\alpha$ together with conv $S$ and the conflict sets $B_{f}, f \in F[S]$, can be found in expected time $O(m)$.

Proof Let $S$ be a random $\alpha$-subset of $Q$. We claim that $S$ is decent with probability at least $1 / 2$. To see this, we first use Lemma A.4(8) with $\gamma=1$ to obtain $\mathbf{E}\left[\sum_{f \in F[S]} b_{f}\right] \ll m$. By Markov's inequality, it follows that $\sum_{f \in F[S]} b_{f} \ll m$ with probability at least 3/4. Furthermore, using Lemma A.2 with $p n=\alpha$ and $t=2 \log \alpha$, we get ${ }^{10} \mathbf{E}\left[\left|F_{\geq 2} \log \alpha\right|\right] \ll\left(\log ^{2} \alpha\right) / \alpha$, and if $\alpha_{0}$ is large enough, this expected value is less than $1 / 4$. Hence, by Markov's inequality, the probability that there exists a facet with conflict size at least $2 m(\log \alpha) / \alpha$ is at most $1 / 4$. So, we have $\max _{f \in F[S]} b_{f} \ll m(\log \alpha) / \alpha$ with probability at least $3 / 4$, and the claimed probability follows from a union bound.

Furthermore, a decent sample can be verified in $O(m)$ time: by the proof of Lemma 4.2, we can find the conflict sets $B_{f}$ and $D_{p}$ in time $O\left(m+\sum_{f \in F[S]} b_{f}\right)$. Hence, we can run the algorithm of Lemma 4.2 on the sample $S$. If the number of steps exceeds $\mathrm{cm}$, for a certain constant $c$ that comes from the proof of Lemma 4.2, we abort the computation and reject the sample, since it cannot be decent. Otherwise, we can check in $O(m)$ time that $\max _{f \in F[S]} b_{f} \ll m(\log \alpha) / \alpha$, as required. Consequently, since a sample is decent with constant probability, repeated sampling yields the desired result. 
Fig. 7 The pruning step: remove all facets at distance at most $2 \lambda$ from a facet with maximum conflict size. The points in $B_{f}^{\prime}, B_{g}^{\prime}$ conflict only with the darker facets at distance at most $\lambda=1$

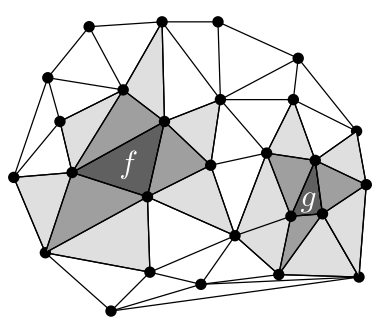

Algorithm 4 Pruning the conflict facets PruneFS

1. Let $\mathcal{F}=\emptyset$, and let queue be a priority queue containing the facets in $F[S]$.

2. While queue $\neq \emptyset$ :

(a) Let $f$ be a facet in queue with maximum $b_{f}^{\prime}$, and let $N_{f}=\left\{f^{\prime} \in F[S] \mid\right.$ $\left.\delta\left(f, f^{\prime}\right) \leq 2 \lambda\right\} \cap$ queue.

(b) Let queue $=$ queue $\backslash N_{f}$ and $\mathcal{F}=\mathcal{F} \cup\{f\}$.

Now let $S$ be a decent sample, and let $B_{f}, f \in F[S]$, denote its conflict sets. By (1) and Property (i) of a decent sample, we have $\sum_{p \in Q} d_{p} \ll m$, and hence there exists a constant $\lambda$ such that the set $X=\left\{p \in Q \mid d_{p}>\lambda\right\}$ has cardinality at most $(1-\mu) m / 2$. Let $R^{\prime}=Q \backslash(S \cup X), B_{f}^{\prime}=B_{f} \cap R^{\prime}$ and $b_{f}^{\prime}=\left|B_{f}^{\prime}\right|$ for $f \in F[S]$. By definition, all points in $R^{\prime}$ conflict with at most $\lambda$ facets. We now prune $F[S]$ to obtain a subset $\mathcal{F}$ of facets whose conflict sets constitute the desired partition. For $f, g \in F[S]$, let $\delta(f, g)$ denote the BFS-distance between $f$ and $g$ in the dual graph of conv $S$; see Fig. 7. ${ }^{11}$ The pruning is done by a greedy algorithm PruneFS, which iteratively takes the facet with the largest conflict size and discards all of its neighbors. For details, see Algorithm 4. Clearly, PruneFS takes $O(m)$ time. Let $f_{1}, \ldots, f_{\beta}$ be the facets in $\mathcal{F}$ as computed by PruneFS, and let $R_{1}, \ldots, R_{\beta}$ be the corresponding conflict sets with respect to $R^{\prime}$. Set $R=\bigcup_{i=1}^{\beta} R_{i}$.

Claim 5.4 We have $|R| \gg m$, the $R_{i}$ constitute a partition of $R$, and for $p \in R_{i}, q \in$ $R_{j}, i \neq j$, we have $D_{p} \cap D_{q}=\emptyset$, and no facet in $D_{p}$ shares an edge with a facet in $D_{q}$.

Proof To see that $|R| \gg m$, note that $\left|N_{f}\right| \ll 1$ and $b_{f^{\prime}}^{\prime} \leq b_{f}^{\prime}$ for every $f^{\prime} \in N_{f}$. Thus, we have $b_{f}^{\prime} \gg \sum_{f^{\prime} \in N_{f}} b_{f^{\prime}}^{\prime}$, and therefore

\footnotetext{
${ }^{10}$ Note that by choosing $\alpha_{0}$ large enough, we can ensure that $t=2 \log \alpha \leq \alpha / 4=p n / 4$.

${ }^{11}$ More precisely, the BFS-distance (BFS = Breadth First Search) between $f$ and $g$ is the length of a shortest path between $f$ and $g$ in the graph with vertex set $F[S]$ in which two vertices are adjacent precisely if the corresponding facets share an edge in conv $S$.
} 


$$
\begin{aligned}
|R| & =\sum_{f \in \mathcal{F}} b_{f}^{\prime} \gg \sum_{f \in \mathcal{F}} \sum_{f^{\prime} \in N_{f}} b_{f^{\prime}}^{\prime} \geq\left|R^{\prime}\right| \geq m-\alpha-(1-\mu) m / 2 \\
& \geq m-\mu m-(1-\mu) m / 2=(1-\mu) m / 2 \gg m .
\end{aligned}
$$

To see that $\left(R_{i}\right)_{1 \leq i \leq \beta}$ is a partition, consider two sets $R_{i}, R_{j}$ with $i \neq j$, and let $f_{i}, f_{j}$ be the corresponding facets. Any point $p \in R$ has $\left|D_{p}\right| \leq \lambda$, and $D_{p}$ is connected in the dual graph of conv $S$. By construction, we have $\delta\left(f_{i}, f_{j}\right)>\lambda$, so there cannot be a point in conflict with both $f_{i}$ and $f_{j}$. It follows that $R_{i} \cap R_{j}=\emptyset$, since $R_{i}$ and $R_{j}$ are the conflict sets of $f_{i}$ and $f_{j}$. Similarly, we see that $D_{p}, D_{q}$ are disjoint for $p \in R_{i}, q \in R_{j}$, and no facet in $D_{p}$ is adjacent to a facet in $D_{q}$, because $\delta\left(f_{i}, f_{j}\right)>2 \lambda$ and $D_{p}, D_{q}$ are connected with size at most $\lambda$.

By now, we have established statements 1-4 of Lemma 5.2. It remains to show how to find all the convex hulls quickly. First, using SplitHull, we can compute the hulls conv $S$, conv $(R \cup S)$, and conv $(Q \backslash(R \cup S))$ in time $O(m)$. It remains to consider the $R_{i}$ 's.

Claim 5.5 For $i=1, \ldots, \beta$, the convex hull conv $R_{i}$ can be computed in $O\left(\left|R_{i}\right|\right)$ time.

Proof Consider an $R_{i}$, and let $f_{i}$ be the corresponding facet in conv $S$. First, note that the subgraph of conv $(R \cup S)$ induced by $R_{i}$ is connected, because $R_{i}=R \cap h_{f_{i}}^{+}$. Let $\Gamma$ denote the points in $(R \cup S) \backslash R_{i}$ that are adjacent in $\operatorname{conv}(R \cup S)$ to a point in $R_{i}$. We have $\Gamma \subseteq S$ : if there were two points $p \in R_{i}, q \in R_{j}, i \neq j$, such that $p q$ is an edge of conv $(R \cup S)$, then $p q$ would also be an edge of conv $(S \cup\{p, q\})$. This implies that either $D_{p} \cap D_{q} \neq \emptyset$ or that there are facets $f^{\prime} \in D_{p}, f^{\prime \prime} \in D_{q}$ such that $f^{\prime}$ and $f^{\prime \prime}$ share an edge. Both are impossible by Claim 5.4.

Next, we claim that $|\Gamma| \ll 1$ : if $p \in R_{i}$ is adjacent to a point $q \in S$, then it follows that $p q$ is also an edge of conv $(S \cup\{p\})$, and hence $D_{p}$ contains a facet incident to $q$. Since $\left|\bigcup_{p \in R_{i}} D_{p}\right| \ll 1$ and since each facet is incident to three points, the claim follows.

Now we compute conv $\left(R_{i} \cup \Gamma\right)$ in $O\left(\left|R_{i}\right|\right)$ time as follows: let $F_{1}$ be the set of facets in $F[R \cup S]$ incident to $R_{i}$, and let $F_{2}$ be the set of facets in $F\left[R_{i} \cup \Gamma\right]$ incident to $R_{i}$. We have $F_{1}=F_{2}$. Clearly, $F_{1} \subseteq F_{2}$ by the definition of $\Gamma$ and since $R_{i} \cup \Gamma \subseteq R \cup S$. If there were a facet $f \in F_{2} \backslash F_{1}$, the halfspace spanned by $f$ would contain only points in $(R \cup S) \backslash\left(R_{i} \cup \Gamma\right)$. However, this would mean that in $\operatorname{conv}(R \cup S)$ all the vertices of $f$ are adjacent to a point in $(R \cup S) \backslash\left(R_{i} \cup \Gamma\right)$, contradicting the choice of $\Gamma$. The facets in $F_{1}$ can be extracted from conv $(R \cup S)$ in time $O\left(\left|R_{i} \cup \Gamma\right|\right)$, and the convex hull of $R_{i} \cup \Gamma$ can be completed in the same time, since the remaining facets involve only points in $\Gamma$, which has constant size. Now conv $R_{i}$ can be extracted from conv $\left(R_{i} \cup \Gamma\right)$ in linear time, either by using SplitHull or by naively removing the points in $\Gamma$ one by one.

This concludes the proof of Lemma 5.2.

Now, the splitting is performed by the algorithm Multisplit. Please refer to Algorithm 5. For the recursion to work, we need to avoid small color classes. 
Algorithm 5 Splitting arbitrary colorings

MultiSplit(conv $P$ )

1. For all colors $i$ with $\left|C_{i}\right| \leq 2^{\sqrt{\log n}}$, find conv $C_{i}$ directly. Let $K$ denote the remaining colors, and $Q=\bigcup_{i \in K} C_{i}$. Use Splithull to determine conv $Q$.

2. Use Lemma 5.2 with $\alpha=2^{\sqrt{\log n}}$ to obtain $S, R \subseteq Q$, a partition of $R_{1}, \ldots, R_{\beta}$ of $R$, and their convex hulls.

3. Call MultiSplit $(\operatorname{conv}(Q \backslash(S \cup R)))$ to find the hulls $\operatorname{conv}\left(C_{i} \cap(Q \backslash(S \cup R))\right)$.

4. For $j=1, \ldots, \beta$, call MultiSplit (conv $\left.R_{j}\right)$ to find the hulls $\operatorname{conv}\left(C_{i} \cap R_{j}\right)$.

5. For $i \in K$, do

(a) For $j=1, \ldots, \beta$, merge $\operatorname{conv}\left(C_{i} \cap R_{j}\right)$ into conv $S$. This yields $\operatorname{conv}(S \cup$ $\left.\left(C_{i} \cap R\right)\right)$.

(b) Use SplitHull to extract $\operatorname{conv}\left(C_{i} \cap(S \cup R)\right)$.

(c) Compute the union of $\operatorname{conv}\left(C_{i} \cap(S \cup R)\right)$ and $\operatorname{conv}\left(C_{i} \cap(Q \backslash(S \cup R))\right)$ to obtain conv $C_{i}$.

Thus, the algorithm first computes the convex hull of every $C_{i}$ with $\left|C_{i}\right| \leq 2^{\sqrt{\log n}}$ in time $O\left(\left|C_{i}\right| \log \left|C_{i}\right|\right)$ [4, Chap. 11]. Let $K$ denote the remaining colors, and let $Q=\bigcup_{i \in K} C_{i}, n_{1}=|Q|$, and $n_{2}=n-n_{1}$. For Step 5(a), we can use an algorithm to combine 3-polytopes separated by a plane [5, Chap. 9.3] to merge each $\operatorname{conv}\left(C_{i} \cap R_{j}\right)$ with $\operatorname{conv}(S)$. For $j \in\{1, \ldots, \beta\}$, this takes time $O\left(1+\left|C_{i} \cap R_{j}\right|\right)$, since all new edges are incident to constantly many points in $S$ by properties 3 and 4 of Lemma 5.2 and since the conflict sets of the $R_{j}$ do not interact. By Theorem 3.1, Step 5(b) takes expected time $O\left(|S|+\left|C_{i} \cap R\right|\right)$, and as Chazelle [11] showed, Step 5(c) needs time $O\left(\left|C_{i}\right|\right)$. Hence, the total expected time for Step 5 is $O\left(|K| \cdot|S|+\sum_{i \in K}\left|C_{i}\right|\right)$. Recall that $\left|C_{i}\right|>2^{\sqrt{\log n}}$ for all $i \in K$. Hence, $|K|<n / 2^{\sqrt{\log n}}$ and $|K| \cdot|S|<n$. Therefore, the total running time of the algorithm is $O\left(n_{2} \sqrt{\log n}+n\right)$, not counting the recursive calls. The first term represents the time for the convex hull computation in Step 1, and the second term counts the remaining steps. We get the following recursion for the running time:

$$
T(n) \leq T(|Q \backslash(S \cup R)|)+\sum_{j=1}^{\beta} T\left(\left|R_{j}\right|\right)+c\left(n_{2} \sqrt{\log n}+n\right)
$$

for some constant $c>0$. We know that $|R| \geq \gamma n_{1}$ and $\max _{1 \leq j \leq \beta}\left|R_{j}\right| \leq c n_{1} \sqrt{\log n} /$ $2^{\sqrt{\log n}}$, where $\gamma \in(0,1]$, and we reuse $c$ (making it larger if necessary). A simple induction shows that $T(n) \ll n \sqrt{\log n}$. Recalling that $|Q|=n_{1}$ and plugging in the inductive hypothesis $T(m) \leq \delta m \sqrt{\log m}$ for $m<n$ and some constant $\delta>0$, we get

$$
\begin{aligned}
T(n) \leq & \delta\left(n_{1}-|R|\right) \sqrt{\log n}+\delta|R| \sqrt{\log n+\log (c \sqrt{\log n})-\sqrt{\log n}} \\
& +c n_{2} \sqrt{\log n}+c n \\
\leq & \delta\left(n_{1}-|R|\right) \sqrt{\log n}+\delta|R| \sqrt{\log n-0.5 \sqrt{\log n}}+c n_{2} \sqrt{\log n}+c n
\end{aligned}
$$


for $n$ large enough. Since $\sqrt{\log n-0.5 \sqrt{\log n}} \leq \sqrt{\log n}-1 / 4$, it follows that

$$
\begin{aligned}
T(n) & \leq \delta\left(n_{1}-|R|\right) \sqrt{\log n}+\delta|R| \sqrt{\log n}-\delta|R| / 4+c n_{2} \sqrt{\log n}+c n \\
& \leq \delta n_{1} \sqrt{\log n}+2 c n_{2} \sqrt{\log n}+(c-\delta \gamma / 4) n_{1},
\end{aligned}
$$

which is bounded by $\delta n \sqrt{\log n}$ for $\delta$ large enough. This concludes the induction and the proof of Theorem 5.1.

\section{Points in Halfspaces}

The problem in this section is to preprocess a point set to report quickly all the points contained in a query halfspace. However, not only do we want to find the points, but also their convex hull. We base our approach on a data structure by Chan [7] that uses filtering search [9]: first, it obtains a superset of the result with comparable size (the candidate set), and then examines each point individually to find the result. By storing not only the candidate sets, but also their convex hulls, we obtain a data structure that reports the convex hull of the points in a query halfspace by using SplitHull. We also show how to improve the preprocessing time over the straightforward $O\left(n \log ^{2} n\right)$.

Theorem 6.1 Let $P \subseteq \mathbb{R}^{3}$ be an $n$-point set in general convex position. In $O(n \log n)$ time we can build a randomized data structure of $O(n \log n)$ size to answer queries of the following kind: given an oriented plane $h$, compute the convex hull of $P \cap h^{+}$, where $h^{+}$denotes the left halfspace of $h$. The expected query time is $O(\log n+k)$, where $k=\left|P \cap h^{+}\right|$denotes the output size.

The main obstacle in improving the preprocessing time is this: given a sample $S \subseteq P$, compute the convex hulls of the conflict sets $B_{f}$ for $f \in F[S]$. In the last section, we modified the conflict sets to obtain a simple algorithm for this problem. This is no longer possible, and we need a more sophisticated approach. Given a plane $h$, let $G(h)$ denote the induced subgraph of conv $P$ with vertex set $P \cap h^{+}$(i.e., $G(h)$ has vertex set $P \cap h^{+}$and contains all edges of conv $P$ with both endpoints in $h^{+}$). Here are some simple facts about $G(h)$ (e.g., [11]); see Fig. 8.

Lemma 6.2 Let $E$ be the set of edges in $G(h)$ incident to a facet of conv $P$ that intersects $h$. There exists a closed walk ${ }^{12} L$ along the edges in $E$ such that $L$ separates $G(h)$ from the rest of conv $P$. Every edge $e \in E$ occurs in $L$ once or twice, depending on whether $e$ is incident to one or two such facets. It follows that $G(h)$ is connected. Given $G(h), L$ can be found in time $O(|V[G(h)]|)$.

Proof Consider the intersection $\mathcal{A}$ of $h$ and conv $P$ (interpreted as a subset of $\mathbb{R}^{3}$ ). The set $\mathcal{A}$ is a two-dimensional convex polygon whose edges correspond to the facets

\footnotetext{
${ }^{12}$ In our terminology, a walk is an arbitrary sequence of adjacent vertices, whereas a path consists of distinct vertices (except possibly the first and the last).
} 
Fig. 8 A lace: $h^{+}$corresponds to the inside of the circle. The lace $L$ is shown in dashed

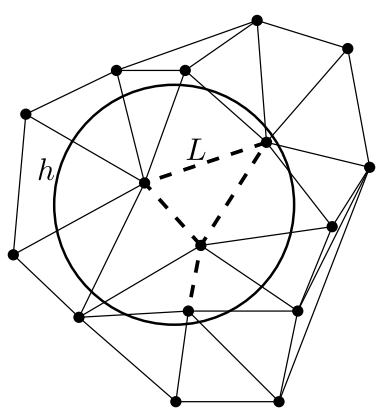

of conv $P$ that intersect $h$. Let $F=f_{1}, f_{2}, \ldots, f_{k}$ be those facets in counterclockwise order along $\mathcal{A}$, and let $F^{\prime}=f_{1}^{\prime}, f_{2}^{\prime}, \ldots, f_{k}^{\prime} \subseteq F$ be the subsequence of facets that are incident to an edge in $E$. Since consecutive facets in $F^{\prime}$ share an incident vertex in $P \cap h^{+}$, the sequence $F^{\prime}$ induces a closed walk $L$ along the edges in $E$. Every path from a point in $P \cap h^{+}$to a point in $P \cap h^{-}$has to cross a point incident to a facet in $F^{\prime}$. Hence, $L$ separates $G(h)$ from the rest of conv $P$. Furthermore, every edge in $E$ appears once in $L$ for each incident facet in $F^{\prime}$. Finally, since consecutive edges in $L$ are consecutive in the cyclic order of edges around their common endpoint in $G(h)$, $L$ can be computed in time linear in the size of $G(h)$.

The walk $L$ is called the lace of $G(h)$; see Fig. 8. Knowing $G(h)$ is enough to compute conv $\left(P \cap h^{+}\right)$quickly.

Corollary 6.3 Given conv $P$ and $G(h)$, we can compute conv $\left(P \cap h^{+}\right)$in time $O\left(\left|P \cap h^{+}\right|\right)$.

Proof The idea is to find an intermediate polytope $\mathcal{P}$ of complexity $O\left(\left|P \cap h^{+}\right|\right)$ whose vertices contain $P \cap h^{+}$. This is done by computing (part of) the intersection of conv $P$ with $h^{+}$and adding a few edges to ensure general position; see Fig. 9. Using SplitHull, we extract conv $\left(P \cap h^{+}\right)$from $\mathcal{P}$ in the desired time.

Let $L$ be the lace of $G(h)$. By Lemma 6.2, $L$ can be found in time $O\left(\left|P \cap h^{+}\right|\right)$ from $G(h)$. Let $F=f_{1}, \ldots, f_{k}$ be the sequence of facets in $F[P]$ that are incident to $L$ and intersect $h$, where the ordering is according to $L$. The sequence $F$ induces in the plane $h$ a sequence $E$ of line segments whose endpoints are in convex position. As the order of $E$ corresponds to the convex hull order, we can compute the convex hull $\mathcal{C}$ of $E$ in linear time. Let $V[\mathcal{C}]$ and $E[\mathcal{C}]$ denote the vertices and edges of $\mathcal{C}$.

We are now ready to construct the convex polytope $\mathcal{P}$. The set of $\mathcal{P}$ 's facets consists of three disjoint parts, $F_{1}, F_{2}$, and $F_{3}$ : (i) $F_{1}$ contains the facets of $G(h)$; (ii) for each line segment $e \in E, F_{2}$ contains a quadrilateral facet spanned by $e$ and its corresponding edge $\tilde{e}$ in $L .{ }^{13}$ Furthermore, for each $e \in E[\mathcal{C}] \backslash E, F_{2}$ contains a triangular facet $f_{e}$ spanned by $e$ and the point in $P \cap h^{+}$incident to the edges whose intersections with $h$ determine $e$; (iii) let $Z$ be the unbounded prism with base $\mathcal{C}$ that extends

${ }^{13}$ That is, $\tilde{e}$ is the edge incident to the facet whose intersections with $h$ create $e$. 


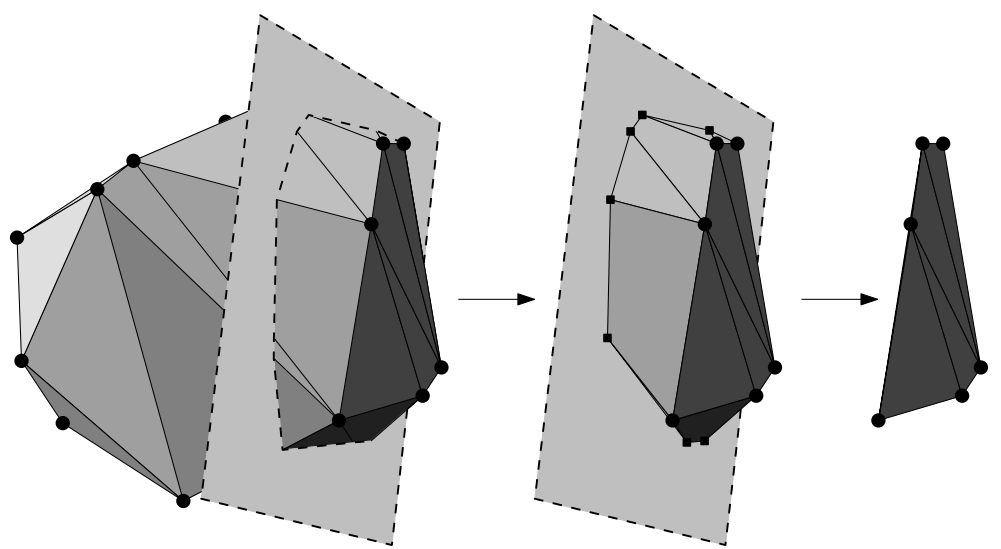

Fig. 9 The halfspace range reporting algorithm: The three stages of Corollary 6.3: Given $G(h)$, find an intermediate polytope that contains the result and split it

Fig. 10 Finding the conflict facets for an edge. $D_{\{p, q\}}$ is darkest, while $D_{p}, D_{q}$ are lighter. $D_{p}$ is bounded by dashed line segments, $D_{q}$ by dotted line segments

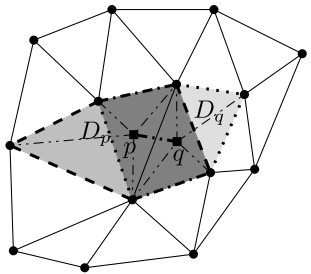

into $h^{-}$. Pick a point $q \in Z \cap$ conv $P$ infinitesimally close to $h . F_{3}$ contains all facets spanned by $q$ and an edge in $E[\mathcal{C}]$. It is easily seen that the facets in $F_{1} \cup F_{2} \cup F_{3}$ are in convex position and bound a convex polytope $\mathcal{P}$ with $O\left(\left|P \cap h^{+}\right|\right)$vertices. Since all the facets of $\mathcal{P}$ have bounded complexity and since all vertices in $V[\mathcal{C}]$ have bounded degree, we can perform a local perturbation of $V[\mathcal{C}]$ to obtain a polytope $\mathcal{P}^{\prime}$ in general position. Now we compute conv $\left(P \cap h^{+}\right)$in time $O\left(\left|V\left[\mathcal{P}^{\prime}\right]\right|\right)=O\left(\left|P \cap h^{+}\right|\right)$ using SplitHull.

For Corollary 6.3, we need to compute all the graphs $G\left(h_{f}\right)$ for $f \in F[S]$ (recall that $h_{f}$ denotes the plane supporting $f$ in $\operatorname{conv} S$ ).

Lemma 6.4 Let $S \subseteq P$ be a random subset. Then the graphs $G\left(h_{f}\right)$ for $f \in F[S]$ can be computed in $O(n)$ expected time.

Proof By Lemma A.4 the total size of the sets $P \cap h_{f}^{+}$and hence the total complexity of the graphs $G\left(h_{f}\right)$ is $O(n)$. Let $e=(p, q) \in E[P]$, and let $D_{e}=D_{p} \cap D_{q}$ be the facets in conflict with both $p$ and $q$; see Fig. 10 . Note that $e \in G\left(h_{f}\right)$ precisely if $f \in D_{e}$. We will compute the sets $D_{e}$ for $e \in E[P]$ and then use them to construct the graphs $G\left(h_{f}\right)$. Let $T_{e}$ denote the graph on vertex set $D_{e}$ where two vertices $f_{1}, f_{2}$ are adjacent if $f_{1}, f_{2}$ share an edge in conv $S$ that is destroyed in conv $(S \cup\{p, q\})$. 
Algorithm 6 Computing the subgraphs

ComputeSubgraphs

1. For every $e \in E[P]$, if $f_{e} \neq \perp$, use $f_{e}$ to compute $D_{e}$. For each $f \in D_{e}$, create records for the two half edges corresponding to $e$ in $G\left(h_{f}\right)$.

2. For every point $p \in P$, use $f_{p}$ to find $D_{p}$. For each $f \in D_{p}$, create a record $p_{f}$ corresponding to $p$ in $G\left(h_{f}\right)$. Every facet in $D_{p}$ has a pointer p which we set to $p_{f}$. For each incident edge $e$ of $p$ in cyclic order, iterate through all facets $f \in D_{e}$. Use the pointer $\mathrm{p}$ of $f$ to find the record $p_{f}$ corresponding to $p$ in $G\left(h_{f}\right)$ and add the appropriate half edge to the edge list of $p_{f}$.

Since $T_{e}$ is connected, ${ }^{14}$ it suffices to compute one facet $f_{e} \in D_{e}$ (if it exists). The remaining facets can be found by traversing $T_{e}$.

We extend SubsetConflictWalk to find conflict facets of edges by changing Step 2(d) as follows: when considering a neighbor $q \in \Gamma_{P}(p)$, we not only compute the conflict facet $f_{q}$, but also a conflict facet $f_{e}$ for the edge $e=\{p, q\}$, if it exists. To do this, let $\Gamma_{p}$ denote the simple polygon in conv $S$ that bounds the conflict region of $p$. The facet $\tilde{f}_{q} \in F[S \cup p]$ is adjacent to an edge $e_{q}$ on $\Gamma_{p}$, and $q$ conflicts with at least one facet in conv $S$ incident to $e_{q}$. Let $f_{1}, f_{2} \in F[S]$ be the facets incident to $e_{q}$, where $f_{1}$ conflicts with $p$ while $f_{2}$ does not. Now, if $q$ conflicts with $f_{1}$, we set $f_{q}=f_{e}=f_{1}$, otherwise, we set $f_{q}=f_{2}$ and $f_{e}=\perp .{ }^{15}$ This takes constant time, and therefore the running time of the algorithm remains linear, as in the proof of Lemma 4.2.

To prove correctness, we claim that if $D_{e} \neq \emptyset$, then $f_{1} \in D_{e}$. Indeed, let $T$ be the graph on vertex set $D_{p} \cup D_{q}$, where two vertices $g_{1}, g_{2}$ of $T$ are adjacent if $g_{1}, g_{2}$ share an edge in $E[S]$ that is destroyed in conv $(S \cup\{p, q\})$. We have that $T_{e}$ is a subgraph of $T$ and that $T$ is a tree (by convex position). Observe that $e_{q}$ corresponds to the edge $e_{q}^{*}=\left\{f_{1}, f_{2}\right\}$ of $T$. Let $T_{1}$ be the connected component of $T \backslash e_{q}^{*}$, with $f_{1} \in T_{1}$. Note that $D_{p} \subseteq V\left[T_{1}\right]$. Furthermore, at least one of $f_{1}, f_{2}$ is in conflict with $q$, hence $D_{q} \cap\left\{f_{1}, f_{2}\right\} \neq \emptyset$. Since the induced subgraph of $T$ on vertex set $D_{q}$ is connected, it follows that if $V\left[T_{1}\right] \cap D_{q} \neq \emptyset$, then $D_{q}$ contains $f_{1}$, and hence $f_{1} \in D_{p} \cap D_{q}=D_{e}$, as desired.

Using the sets $D_{e}$, we can now compute a DCEL representation of the graphs $G\left(h_{f}\right)$ in $O(n)$ time through careful pointer manipulation (Algorithm 6).

Proof of Theorem 6.1 We rely on a variant of Chan's data structure [7] due to Ramos [31]. The candidate sets are the conflict sets of an appropriate gradation of $P$. By Corollary 6.3 and Lemma 6.4, we can find their convex hulls in time $O(n \log n)$. To process a query, we extend the original query algorithm to use SplitHull on the candidate set after coloring the points in $h^{+}$blue.

The details are as follows: take a gradation $\emptyset=P_{-1} \subseteq P_{0} \subseteq \cdots \subseteq P_{\log n}=P$, where $P_{i-1}$ is derived from $P_{i}$ by sampling every point with probability $1 / 2$. We

\footnotetext{
${ }^{14}$ We define the empty graph to be connected.

${ }^{15} \mathrm{As}$ is often done in the study of programming languages, we use $\perp$ as a symbol for an undefined value.
} 
compute the convex hulls conv $P_{i}$ in time $O(n \log n)$. Using Lemma 6.4 and Corollary 6.3, we then find the convex hulls conv $B_{f}$ for all the conflict sets $B_{f}, f \in F\left[P_{i}\right]$, $i=0, \ldots, \log n$. Since this takes $O(n)$ time for each $i$, the total time is $O(n \log n)$. Now we switch into dual space. For this, we use duality with respect to the unit paraboloid which turns upper convex hulls into upper envelopes and lower convex hulls into lower envelopes [30, Chap. 2.4.1]. We compute two data structures, one for the upper envelope and one for the lower envelope, focusing the discussion on the lower envelope. For each $i=0, \ldots, \log n$, we find the set of planes $H_{i}$ dual to $P_{i}$ and a canonical triangulation $T_{i}$ of the lower envelope of $H_{i}$ (this takes linear time since we know conv $P_{i}$ ). Then we construct a point location structure for the $x y$-projection of $T_{i}$. Every facet $\Delta$ of $T_{i}$ is incident to at most three points of the lower envelope of $H_{i}$, corresponding to at most three facets $f_{1}, f_{2}, f_{3}$ of conv $P_{i}$. Let $B_{\Delta}=B_{f_{1}} \cup B_{f_{2}} \cup B_{f_{3}}$. We compute conv $B_{\Delta}$ in linear time [11] and store it with $\Delta$. By the properties of canonical triangulations and the arguments given by Chan [7], the preprocessing phase takes expected time $O(n \log n)$ and uses expected space $O(n \log n)$. Then we repeat the process to obtain two independent data structures $D_{1}, D_{2}$.

Now suppose that we are given a query plane $h$. We need to find all the planes in $H$ below $h^{*}$, the point dual to $h$. Let $\ell$ be the vertical line through $h^{*}$. Perform the following procedure simultaneously on $D_{1}$ and $D_{2}$, until one of them yields the answer: For $i=\log (n / \log n), \log (n / \log n)-1, \ldots, 0$, locate the facet $\Delta_{i}$ of $T_{i}$ intersected by $\ell$ in $O(\log n)$ time with the point location structure. Stop when the dual point $h^{*}$ lies below the lower envelope of $H_{i}$. Now find the planes in $H$ below $h^{*}$ by inspecting the conflict set $B_{\Delta_{i}}$ and use SplitHull to compute conv $\left(P \cap h^{+}\right)$in $O\left(\left|B_{\Delta_{i}}\right|\right)$ time. As was argued by Ramos [31, Sect. 2.2.1], such a query takes expected time $O\left(\log n+\left|P \cap h^{+}\right|\right)$, as claimed. For completeness, we have included the calculation in Appendix B.

\section{Union of Hulls}

Finally, we consider the issue of output-sensitivity: if we are only interested in the hull of the blue points, under which circumstances can it be computed quickly without looking at the whole polytope? For this, we look at the problem DisJUniON, where the task is the following: given point sets $P_{1}, \ldots, P_{k} \subseteq \mathbb{R}^{3}$ and their convex hulls conv $P_{1}, \ldots$, conv $P_{k}$ such that conv $P_{i} \cap \operatorname{conv} P_{j}=\varnothing$ for $i \neq j$ and such that $P=$ $\bigcup_{i=1}^{k} P_{i}$ is in convex position, we would like to compute conv $P$. In general, we cannot do better than to repeatedly merge pairs of the hulls.

Theorem 7.1 Any algorithm that solves DISJUNION requires $\Omega(|P| \log k)$ comparisons.

Proof We use an old lower bound [11, Sect. 4A] and combine it with Seidel's method of including the index as a coordinate [32]. We reduce from the list merging problem, in which $k$ sorted lists of numbers need to be merged into one. We lift the lists onto the unit paraboloid $y=x^{2}$, using the $z$-coordinate to represent the index of the list. 
Fig. 11 An illustration of the reduction from LISTMERGE to DISJUNION for the 3 lists $(5,9,12,14),(1,8)$, $(2,4,6,7,10,13)$, and $(3,11)$. The path marked by the bold edges represents the merged list

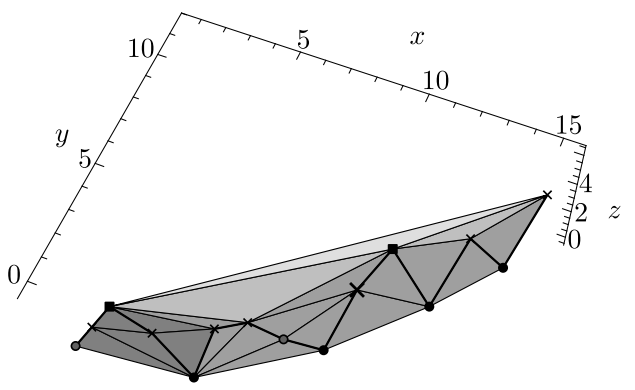

Clearly, the lifting and the individual convex hulls, which are pairwise disjoint, can be found in time $O(n)$. A simple geometric argument now shows that the merged list can be derived from the convex hull of the union in linear time; see Fig. 11.

More precisely, consider the problem LISTMERGE: given $k$ sorted integer sequences $L_{1}, \ldots, L_{k}$, compute the sorted list $L=\bigcup_{i=1}^{k} L_{i}$. A straightforward counting argument shows that any algorithm for LISTMERGE requires $\Omega(|L| \log k)$ comparisons. We describe a linear time reduction from ListMERGE to DisJUNion: let $L_{i}=\left(r_{1}, \ldots, r_{j}\right)$. We map $L_{i}$ to a point set $P_{i} \subseteq \mathbb{R}^{3}$ by mapping each $r_{z}$ to $p\left(r_{z}\right)=\left(r_{z}, r_{z}^{2}, i\right)$. All the points lie on the parabolic surface $y=x^{2}$, and hence $P=\bigcup_{i=1}^{k} P_{i}$ is in convex position. Furthermore, each $P_{i}$ is contained in the plane $z=i$, and hence conv $P_{i} \cap \operatorname{conv} P_{j}=\emptyset$ for $i \neq j$. The conv $P_{i}$ can be computed in linear time, since the lists $L_{i}$ are sorted.

If $r, s$ are consecutive in the sorted list $L$, then $p(r) p(s)$ is an edge of conv $P$. To see this, let $\hat{p}(r), \hat{p}(s)$ denote the projections of $p(r), p(s)$ onto the $x y$-plane, and let $h$ denote the plane orthogonal to the $x y$-plane that contains the line segment $\hat{p}(r) \hat{p}(s)$. By definition, $h$ contains $p(r)$ and $p(s)$, and hence also the line segment $p(r) p(s)$. Furthermore, all other points of $P$ are on the same side of $h$. For this, fix $i \in\{1, \ldots, k\}$ and consider the parabola $Z_{i}: x \mapsto\left(x, x^{2}, i\right)$. Clearly, $h$ intersects $Z_{i}$ in the points $\left(r, r^{2}, i\right)$ and $\left(s, s^{2}, i\right)$, cutting off the part of $Z_{i}$ between $r$ and $s$. Since $r$ and $s$ are consecutive in $L$, this part contains no points in $P_{i}$. It follows that $h$ supports the line segment $p(r) p(s)$, making it an edge of conv $P$. Consequently, it takes $\Omega(|P| \log k)$ time to compute conv $P$, since otherwise we could recover the sorted list $L$ by examining the $O(|P|)$ edges of conv $P$.

Intuitively, what makes our lower bound instance hard is the fact that when merging conv $P_{i}$, we need to switch often between the individual hulls in an unpredictable way. We can avoid this by imposing additional constraints on the input, and thus obtain a better result.

Theorem 7.2 Let $Q \subseteq \mathbb{R}^{3}$ be in general convex position. Let $P=\bigcup_{i=1}^{k} P_{i} \subseteq Q$ with $|P|=n$ such that the $P_{i}$ are pairwise disjoint and the subgraphs conv $\left.Q\right|_{P_{i}}$ are connected. Then, given spanning trees $T_{1}, \ldots, T_{k}$ for conv $\left.Q\right|_{P_{i}}$, we can compute conv $P$ in expected time $O\left(n \log ^{*} n+k \log k\right)$.

Proof We use Seidel's tracing technique [33]: pick a subset $K \subseteq P$ that meets each $T_{i}$ in exactly one point, and an appropriate gradation $S_{0} \subseteq \cdots \subseteq S_{\beta}=P \backslash K$ with 
$\beta \ll \log ^{*} n$. Then compute conv $\left(S_{0} \cup K\right)$ in time $O(n+k \log k)$ and successively each conv $\left(S_{i} \cup K\right)$ in $O(n)$. Here, the bottleneck is to locate the conflict facets for $S_{i+1}$ in $\operatorname{conv}\left(S_{i} \cup K\right)$. This is done using the spanning trees $T_{i}$ and an appropriate variant of SubsetConflictWalk.

We may assume that $k<n / 2$, since otherwise the theorem is easy. Let $K \subseteq P$ be such that $K$ contains exactly one point of each $P_{i}$, and let $m=n-k$. Let $z=\max \{k, m / \log m\}$ and choose $1 \leq \alpha \leq \log ^{*} m$ such that $m / \log ^{(\alpha-1)} m<z \leq$ $m / \log ^{(\alpha)} m$, where $\log ^{(i)} m$ denotes the $i$ th iterated $\operatorname{logarithm}{ }^{16}$ of $m$. Let $\beta=$ $\log ^{*} m-\alpha+1$. Compute a gradation of subsets $S_{0} \subseteq \cdots \subseteq S_{\beta}=P \backslash K$ such that $S_{i}$ is a random subset of $S_{i+1}$ with $\left|S_{0}\right|=z$ and $\left|S_{i+1}\right|=\left|S_{i}\right| \log ^{(\alpha+i)} \mathrm{m} / \log ^{(\alpha+i+1)} \mathrm{m}$ for $0 \leq i<\beta$. By induction, it follows that $\left|S_{i}\right| \leq m / \log ^{(\alpha+i)}$. For $i=0, \ldots, \beta$, let $\widetilde{S}_{i}=S_{i} \cup K$. We will show how to compute conv $\widetilde{S}_{i+1}$ from conv $\widetilde{S}_{i}$ in time $O(n)$ for each $i$. Furthermore, conv $\widetilde{S}_{0}$ can be computed in time $O(n+k \log k)$ with a regular convex hull algorithm, as $\left|S_{0} \cup K\right|=O(n / \log n+k)$. Hence, it takes $O\left(n \log ^{*} n+k \log k\right)$ steps to compute conv $Q=\operatorname{conv} \widetilde{S}_{\beta}$.

To derive conv $\widetilde{S}_{i+1}$ from conv $\widetilde{S}_{i}$, we proceed in two steps: first, we determine the conflict sets $B_{f}$ for $f \in F\left[\widetilde{S}_{i}\right]$. Below, we will argue that this can be done in linear time. Then, we use the algorithm from Lemma A.5 to compute conv $\widetilde{S}_{i+1}$. This takes time proportional to

$$
\begin{aligned}
\left(\left|S_{i+1}\right|+k \frac{\left|S_{i+1}\right|}{\left|S_{i}\right|}\right) \log \frac{\left|S_{i+1}\right|}{\left|S_{i}\right|} & \leq 2\left|S_{i+1}\right| \log \left(\frac{\log ^{(\alpha+i)} m}{\log ^{(\alpha+i+1)} m}\right) \\
& \ll \frac{m}{\log ^{(\alpha+i+1)} m} \log \left(\frac{\log ^{(\alpha+i)} m}{\log ^{(\alpha+i+1)} m}\right),
\end{aligned}
$$

since $k \leq\left|S_{0}\right| \leq\left|S_{i}\right|$. The last term is $O(n)$, as claimed.

It remains to show how to find the conflict sets $B_{f}$ in time $O(n)$. For each $j=1, \ldots, k$, we determine conflict facets for $P_{j}$ as follows: let $r_{j}=P_{j} \cap K$. We use a slight modification of SubsetConflictWalk: merge the neighbors of $r_{j}$ in conv $\widetilde{S}_{i}$ with the neighbors $\Gamma_{T_{j}}\left(r_{j}\right)$ of $r_{j}$ in $T_{j}$ in order to find a conflict facet $f_{p}$ for each $p \in \Gamma_{T_{j}}\left(r_{j}\right)$. Then continue in a BFS-manner along $T_{j}$, inserting in turn each $p \in \Gamma_{T_{j}}\left(r_{j}\right)$ into conv $\widetilde{S}_{i}$, and so on. As in Sect. 4, we see that the total time is proportional to

$$
\begin{aligned}
\sum_{p \in \widetilde{S}_{i}} \operatorname{deg}_{\widetilde{S}_{i}} p+\sum_{j=1}^{k} \sum_{p \in T_{j}} \operatorname{deg}_{T_{j}} p+\sum_{p \in P \backslash S_{i}} d_{p} & \ll\left|\widetilde{S}_{i}\right|+|P|+\sum_{f \in F\left[\widetilde{S}_{i}\right]} b_{f} \\
& \ll|P|+n-k+k \frac{\left|S_{i+1}\right|}{\left|S_{i}\right|},
\end{aligned}
$$

by Lemma A.4. Since $k \leq\left|S_{i}\right|$ and $\left|S_{i+i}\right| \leq n$, the last term is linear. This finishes the proof.

${ }^{16}$ Defined by $\log \left({ }^{(0)} m=m\right.$ and $\log (k) m=\max \{1, \log (\log (k-1) m)\}$ for $k \geq 1$. 
For our original question, this means that we can quickly compute the blue hull without considering the whole polytope, as long as the number of induced blue components is small.

Corollary 7.3 Let $P \subseteq \mathbb{R}^{3}$ be a finite point set in general convex position, and let $B$ be a subgraph of conv $P$ with $n$ vertices. Then conv $V[B]$ can be computed in time $O\left(n \log ^{*} n+k \log k\right)$, where $k$ denotes the number of connected components of $B$.

Proof This follows immediately from Theorem 7.2. Given $B$, we can find spanning trees for its components in $O(n)$ time, using, say, depth-first search [20].

In particular, we get the following nice fact about Delaunay triangulations, which provides a Delaunay analogue to an old result by Bar-Yehuda and Chazelle [3].

Corollary 7.4 Let $T=(V, E)$ be a Delaunay triangulation, and let $S \subseteq T$ be a set of $n$ vertices and edges of $T$ with $k$ connected components. Then the Delaunay triangulation of $S$ can be computed in time $O\left(n \log ^{*} n+k \log k\right)$.

Proof Use Corollary 7.3 and the connection between planar Delaunay triangulations and three-dimensional convex hulls [4, Chap. 11.4].

\section{Appendix A: Clarkson-Shor Toolbox}

We review a few tools from geometric random sampling theory [19, 30]. Our presentation follows Ramos [31]. Let $P \subseteq \mathbb{R}^{3}$ with $|P|=n$, and $K \subseteq P$ with $|K|=k$. Given a triple $\mathbf{u}=\left(p_{1}, p_{2}, p_{3}\right) \in P^{3}$, let $h_{\mathbf{u}}$ be the plane spanned by $\mathbf{u}$, oriented such that the set of vectors $\left\{u_{2}-u_{1}, u_{3}-u_{1}, p-u_{1}\right\}$ has positive determinant for $p \in h_{\mathbf{u}}^{+}$. A point $p \in P$ conflicts with $\mathbf{u}$ if $p$ lies in $h_{\mathbf{u}}^{+}$. Let $B_{\mathbf{u}}$ denote the set of all points in $P$ that conflict with $\mathbf{u}$, and $b_{\mathbf{u}}=\left|B_{\mathbf{u}}\right|$.

Lemma A.1 Fix $p \in(0,1]$ and $t \geq 1$. Let $S \subseteq P \backslash K$ be a random subset of size $p(n-k)$, and let $S^{\prime} \subseteq P \backslash K$ be a random subset of size $p^{\prime}(n-k)$ for $p^{\prime}=p / t$. Suppose that $p^{\prime}(n-k) \geq 4$. Fix $\boldsymbol{u}=\left(p_{1}, p_{2}, p_{2}\right) \in P^{3}$, and let $f_{\boldsymbol{u}}$ be the facet defined by $\boldsymbol{u}$. Then

$$
\operatorname{Pr}\left[f_{\boldsymbol{u}} \in F[S \cup K]\right] \ll t^{3} \exp \left(-\frac{(t-1) p b_{\boldsymbol{u}}}{t}\right) \operatorname{Pr}\left[f_{\boldsymbol{u}} \in F\left[S^{\prime} \cup K\right]\right] .
$$

Proof Let $\sigma=\operatorname{Pr}\left[f_{\mathbf{u}} \in F[S \cup K]\right]$ and $\sigma^{\prime}=\operatorname{Pr}\left[f_{\mathbf{u}} \in F\left[S^{\prime} \cup K\right]\right]$. Note that $f_{\mathbf{u}}$ appears in $F[S \cup K]$ precisely if $\mathbf{u} \subseteq S \cup K$ and $B_{\mathbf{u}} \cap(S \cup K)=\emptyset$. If $K \cap B_{\mathbf{u}} \neq \emptyset$, then $\sigma=\sigma^{\prime}=0$, and the lemma holds. Thus, we may assume that $K$ and $B_{\mathbf{u}}$ are disjoint. Let $m=n-k$, and let $d_{\mathbf{u}}$ denote $3-|K \cap \mathbf{u}|$, the number of points in $\mathbf{u}$ not in $K$. Since there are $\left(\begin{array}{c}m-b_{\mathbf{u}}-d_{\mathbf{u}} \\ p m-d_{\mathbf{u}}\end{array}\right)$ ways of choosing a $p m$-subset from $P \backslash K$ that avoids all elements in $B_{\mathbf{u}}$ and contains all the relevant points of $\mathbf{u}$, we have

$$
\sigma=\left(\begin{array}{c}
m-b_{\mathbf{u}}-d_{\mathbf{u}} \\
p m-d_{\mathbf{u}}
\end{array}\right) /\left(\begin{array}{c}
m \\
p m
\end{array}\right)
$$




$$
\begin{aligned}
& =\frac{\prod_{j=0}^{p m-d_{\mathbf{u}}-1}\left(m-b_{\mathbf{u}}-d_{\mathbf{u}}-j\right)}{\prod_{j=0}^{p m-d_{\mathbf{u}}-1}\left(p m-d_{\mathbf{u}}-j\right)} / \frac{\prod_{j=0}^{p m-1}(m-j)}{\prod_{j=0}^{p m-1}(p m-j)} \\
& =\prod_{j=0}^{d_{\mathbf{u}}-1} \frac{p m-j}{m-j} \prod_{j=0}^{p m-d_{\mathbf{u}}-1} \frac{m-b_{\mathbf{u}}-d_{\mathbf{u}}-j}{m-d_{\mathbf{u}}-j} \\
& \leq p^{d_{\mathbf{u}}} \prod_{j=0}^{p m-d_{\mathbf{u}}-1}\left(1-\frac{b_{\mathbf{u}}}{m-d_{\mathbf{u}}-j}\right) .
\end{aligned}
$$

Similarly, we get

$$
\sigma^{\prime}=\prod_{j=0}^{d_{\mathbf{u}}-1} \frac{p^{\prime} m-j}{m-j} \prod_{j=0}^{p^{\prime} m-d_{\mathbf{u}}-1}\left(1-\frac{b_{\mathbf{u}}}{m-d_{\mathbf{u}}-j}\right),
$$

and since $p^{\prime} m \geq 4$ and $j \leq 2$ (in the first product), it follows that

$$
\sigma^{\prime} \geq\left(\frac{p^{\prime}}{2}\right)^{d_{\mathbf{u}}} \prod_{j=0}^{p^{\prime} m-d_{\mathbf{u}}-1}\left(1-\frac{b_{\mathbf{u}}}{m-d_{\mathbf{u}}-j}\right) .
$$

Therefore, since $p^{\prime}=p / t$,

$$
\begin{aligned}
\frac{\sigma}{\sigma^{\prime}} & \leq 8\left(\frac{p}{p^{\prime}}\right)^{d_{\mathbf{u}}} \prod_{j=p^{\prime} m-d_{\mathbf{u}}}^{p m-d_{\mathbf{u}}-1}\left(1-\frac{b_{\mathbf{u}}}{m-d_{\mathbf{u}}-j}\right) \leq 8 t^{3}\left(1-\frac{b_{\mathbf{u}}}{m}\right)^{(t-1) p m / t} \\
& \leq 8 t^{3} \exp \left(-\frac{(t-1) p b_{\mathbf{u}}}{t}\right)
\end{aligned}
$$

as desired.

The lemma implies a Chernoff-type bound for the conflict size of a random sample.

Lemma A.2 Fix $p \in(0,1]$ and let $S \subseteq P$ be a random subset of size pn. Fix $t \geq 1$ such that $t \leq p n / 4$ and let $F_{\geq t}=\left\{f \in F[S] \mid b_{f} \geq t / p\right\}$. Then

$$
\boldsymbol{E}\left[\left|F_{\geq t}\right|\right] \ll t^{2} e^{-t} p n .
$$

Proof Let $S^{\prime} \subseteq P$ be a random subset of size $p n / t$. Since $p n / t \geq 4$, we have

$$
\begin{aligned}
\mathbf{E}\left[\left|F_{\geq t}\right|\right] & =\sum_{\substack{\mathbf{u} \in P^{3} \\
b_{\mathbf{u} \geq t / p}}} \operatorname{Pr}\left[f_{\mathbf{u}} \in F[S]\right] \\
& \ll \sum_{\substack{\mathbf{u} \in P^{3} \\
b_{\mathbf{u} \geq t / p}}} t^{3} \exp \left(-\frac{(t-1) p b_{\mathbf{u}}}{t}\right) \operatorname{Pr}\left[f_{\mathbf{u}} \in F\left[S^{\prime}\right]\right]
\end{aligned}
$$




$$
\ll t^{3} e^{-t} \mathbf{E}\left[\left|F\left[S^{\prime}\right]\right|\right] \ll t^{2} e^{-t} p n,
$$

because $\mathbf{E}\left[\left|F\left[S^{\prime}\right]\right|\right] \ll p n / t$.

Next, we want to bound the average conflict size. For this, we first determine the average for a particular function, from which we then deduce bounds for a large class of well-behaved functions.

Lemma A.3 Fix $p \in(0,1]$ and let $S \subseteq P \backslash K$ be a random subset of size $p(n-k)$. Then

$$
\boldsymbol{E}\left[\sum_{f \in F[S \cup K]} \exp \left(\frac{p b_{f}}{2}\right)\right] \ll p(n-k)+k .
$$

Proof We may assume that $p(n-k) / 2 \geq 4$, because otherwise $p b_{f}=O(1)$ for every $f \in F[S \cup K]$ (as all these $f$ have $b_{f} \leq n-k$ and $\operatorname{conv}(S \cup K)$ has $O(p(n-k)+k)$ facets), and the lemma would hold trivially. Let $S^{\prime} \subseteq P \backslash K$ be a random subset of size $p(n-k) / 2$. We have

$$
\begin{aligned}
\mathbf{E}\left[\sum_{f \in F[S \cup K]} \exp \left(\frac{p b_{f}}{2}\right)\right] & =\sum_{\mathbf{u} \in P^{3}} \operatorname{Pr}\left[f_{\mathbf{u}} \in F[S \cup K]\right] \exp \left(\frac{p b_{\mathbf{u}}}{2}\right) \\
& \ll \sum_{\mathbf{u} \in P^{3}} \operatorname{Pr}\left[f_{\mathbf{u}} \in F\left[S^{\prime} \cup K\right]\right] \\
& =\mathbf{E}\left[\left|F\left[S^{\prime} \cup K\right]\right|\right] \ll p(n-k)+k .
\end{aligned}
$$

Using this bound, we can show that the sum of every well-behaved function over the conflict sizes of a random sample gives the value one would expect. This remains true if a few points from $P$ are always included in the sample.

Lemma A.4 Fix $p \in(0,1]$ and let $S \subseteq P \backslash K$ be a random subset of size $p(n-k)$. Let $g$ be a function such that $g(t n) \ll e^{t} g(n)$ for all $t \geq 0$. Then

$$
\boldsymbol{E}\left[\sum_{f \in F[S \cup K]} g\left(b_{f}\right)\right] \ll(p(n-k)+k) g(1 / p) .
$$

In particular, choosing $k=0$ and $g: n \mapsto n^{\gamma}$ for $\gamma \geq 0$, we have

$$
\boldsymbol{E}\left[\sum_{f \in F[S]} b_{f}^{\gamma}\right] \ll n p^{1-\gamma},
$$

and choosing $g: n \mapsto n \log n$, we get

$$
\boldsymbol{E}\left[\sum_{f \in F[S \cup K]} b_{f} \log b_{f}\right] \ll(n-k+k / p) \log (1 / p) .
$$


Proof We have

$$
\begin{aligned}
\mathbf{E}\left[\sum_{f \in F[S \cup K]} g\left(b_{f}\right)\right] & =\mathbf{E}\left[\sum_{f \in F[S \cup K]} g\left(\frac{p b_{f}}{2} \cdot \frac{2}{p}\right)\right] \\
& \ll e^{2} g(1 / p) \mathbf{E}\left[\sum_{f \in F[S \cup K]} \exp \left(p b_{f} / 2\right)\right] \\
& \ll(p(n-k)+k) g(1 / p),
\end{aligned}
$$

by (7).

The following lemma follows from a standard application of the geometric divideand-conquer technique $[12,18,19]$ and asserts that a convex hull can be computed faster if a random partial hull and the corresponding conflict information are known.

Lemma A.5 Fix $p \in(0,1]$ and let $S \subseteq P \backslash K$ be a subset of size $p(n-k)$. Suppose that conv $(S \cup K)$ and the conflict sets $B_{f} \subseteq P$ for $f \in F[S \cup K]$ are available. Then we can find conv $P$ in expected time $\sum_{f \in F[S \cup K]} b_{f} \log b_{f}$. In particular, if $S$ is a random subset, the running time is $O((n-k+k / p) \log (1 / p))$.

Proof Let $\widetilde{S}=S \cup K$. Without loss of generality, we assume that conv $\widetilde{S}$ contains the origin. Instead of conv $P$, we compute $\left(P^{*}\right)^{\bigcap}$, the intersection of the halfspaces dual to the points in $P$. For this, we first obtain $\left(\widetilde{S}^{*}\right)^{\cap}$, which takes linear time, since conv $\widetilde{S}$ is known. The vertices of $\left(\widetilde{S}^{*}\right)^{\cap}$ correspond to the facets of conv $\widetilde{S}$. In particular, each vertex $f$ of $\left(\widetilde{S}^{*}\right)^{\cap}$ has a conflict list $B_{f}^{*}$ of size $b_{f}$. We compute a tetrahedralization $\mathcal{T}$ of $\left(\widetilde{S}^{*}\right)^{\cap}$ as follows: for each facet $g$ of $\left(\widetilde{S}^{*}\right)^{\bigcap}$, determine the vertex $f_{g}$ incident to $g$ with minimum $b_{f_{g}}{ }^{17}$ The vertex $f_{g}$ is called the apex of $g$. Triangulate $g$ by adding line segments from the apex to all other vertices of $g$. Finally, extend this triangulation to a tetrahedralization by lifting it to the origin. This takes linear time.

The conflict set of a simplex $s$ is precisely $B_{s}^{*}=B_{f_{1}}^{*} \cup B_{f_{2}}^{*} \cup B_{f_{3}}^{*}$, where $f_{1}, f_{2}$, $f_{3}$ are the vertices of $s$ other than the origin. Let $b_{s}=\left|B_{s}\right|$. We determine the intersection of the halfspaces in $B_{s}^{*}$ and clip it to $s$. Then we glue the parts together to obtain $\left(P^{*}\right)^{\cap}$ and hence conv $P$. This takes time $O\left(\sum_{s \in \mathcal{T}} b_{s} \log b_{s}\right)$. Consider a simplex $s \in \mathcal{T}$ and let $f_{s}, f_{1}, f_{2}$ be its vertices other than the origin. Here $f_{s}$ denotes the apex of the facet of $\left(\widetilde{S}^{*}\right)^{\cap}$ that contains a facet of $s$, and we call $f_{s}$ also the apex of $s$. By definition, we have $b_{s}=f_{s}+f_{1}+f_{2} \leq 2\left(f_{1}+f_{2}\right)$, and hence $b_{s} \log b_{s} \ll f_{1} \log f_{1}+f_{2} \log f_{2}$. By general position, every vertex of $\left(\widetilde{S}^{*}\right)^{\cap}$ has degree 3 and thus appears in only constantly many simplices of $\mathcal{T}$ as a nonapex. Hence, $\sum_{s \in \mathcal{T}} b_{s} \log b_{s} \ll \sum_{f \in F[\widetilde{S}]} b_{f} \log b_{f}$, as claimed. Now, if $S$ is a random sample, this sum is proportional to $(n-k+k / p) \log (1 / p)$, by Corollary A.4(9).

${ }^{17}$ We take the lexicographically smallest if there is more than one such vertex. 


\section{Appendix B: Analyzing the Halfspace Range Reporting Structure}

We present Ramos' analysis [31] of a variant of Chan's data structure [7]. Let $\mathcal{E}_{i}$ denote the event that $i$ is the largest index for which $h^{*}$ lies below the lower envelope of $H_{i}$ in either $D_{1}$ or $D_{2}$. The expected running time is

$$
\sum_{i=0}^{\log n-\log \log n}\left((\log n-\log \log n-i) \log n+\mathbf{E}\left[\left|B_{\Delta_{i}}\right| \mid \mathcal{E}_{i}\right]\right) \operatorname{Pr}\left[\mathcal{E}_{i}\right] .
$$

Let $k=\left|P \cap h^{+}\right|$. Note that $\mathbf{E}\left[\left|B_{\Delta_{i}}\right| \mid \mathcal{E}_{i}\right]=O\left(k+n / 2^{i}\right)$, since by standard random sampling theory the expected size of a conflict list in $T_{i}$ is $O\left(n / 2^{i}\right)$ and the random choices for the points in $P \backslash\left(P \cap h^{+}\right)$are independent of $\mathcal{E}_{i}$. Thus,

$$
\begin{aligned}
(10) & \ll \sum_{i=0}^{\log (n / \log n)}\left(\left(\log \left(\frac{n}{\log n}\right)-i\right) \log n+k+\frac{n}{2^{i}}\right) \operatorname{Pr}\left[\mathcal{E}_{i}\right] \\
& \ll k+\sum_{i=1 \log (n / k)}^{\log (n / \log n)}\left(\left(\log \left(\frac{n}{\log n}\right)-i\right) \log n+\frac{n}{2^{i}}\right) \operatorname{Pr}\left[\mathcal{E}_{i}\right] \\
& +\sum_{i=0}^{\log (n / k)-1}\left(\left(\log \left(\frac{n}{\log n}\right)-i\right) \log n+\frac{n}{2^{i}}\right) \operatorname{Pr}\left[\mathcal{E}_{i}\right] .
\end{aligned}
$$

If $k<\log n$, the first sum is zero. Otherwise, we get

$$
\begin{aligned}
& \sum_{i=\log (n / k)}^{\log (n / \log n)}\left(\left(\log \left(\frac{n}{\log n}\right)-i\right) \log n+\frac{n}{2^{i}}\right) \operatorname{Pr}\left[\mathcal{E}_{i}\right] \\
& \leq(\log n) \log \left(\frac{k}{\log n}\right)+\sum_{i \geq \log (n / k)} \frac{n}{2^{i}} \ll k
\end{aligned}
$$

To bound the second sum, we observe that $\operatorname{Pr}\left[\mathcal{E}_{i}\right] \leq\left(k 2^{i+1} / n\right)^{2}$, because if $\mathcal{E}_{i}$ holds, then $P_{i+1}$ in both data structures $D_{1}$ and $D_{2}$ must necessarily contain one of the at most $k$ points in $P \cap h^{+}$. We get

$$
\begin{aligned}
& \sum_{i=0}^{\log (n / k)-1}\left(\left(\log \left(\frac{n}{\log n}\right)-i\right) \log n+\frac{n}{2^{i}}\right) \operatorname{Pr}\left[\mathcal{E}_{i}\right] \\
& \leq(\log n) \log \left(\frac{k}{\log n}\right)+\sum_{i=0}^{\log (n / k)-1}\left(\left(\log \left(\frac{n}{k}\right)-i\right) \log n+\frac{n}{2^{i}}\right)\left(\frac{k 2^{i+1}}{n}\right)^{2} \\
& \ll k+\sum_{j=1}^{\log (n / k)}\left(j \log n+2^{j} k\right) 2^{-2(j-1)} \ll \log n+k
\end{aligned}
$$

as desired. 


\section{References}

1. Aggarwal, A., Guibas, L.J., Saxe, J., Shor, P.W.: A linear-time algorithm for computing the Voronoi diagram of a convex polygon. Discrete Comput. Geom. 4(6), 591-604 (1989)

2. Amato, N.M., Goodrich, M.T., Ramos, E.A.: Linear-time triangulation of a simple polygon made easier via randomization. In: Proc. 16th Annu. ACM Sympos. Comput. Geom. (SoCG), pp. 201-212 (2000)

3. Bar-Yehuda, R., Chazelle, B.: Triangulating disjoint Jordan chains. Int. J. Comput. Geom. Appl. 4(4), 475-481 (1994)

4. de Berg, M., Cheong, O., van Kreveld, M., Overmars, M.: Computational Geometry: Algorithms and Applications, 3rd edn. Springer, Berlin (2008)

5. Boissonnat, J.D., Yvinec, M.: Algorithmic Geometry. Cambridge University Press, New York (1998)

6. Buchin, K., Mulzer, W.: Delaunay triangulations in $O(\operatorname{sort}(n))$ time and more. In: Proc. 50th Annu. IEEE Sympos. Found. Comput. Sci. (FOCS), pp. 139-148 (2009)

7. Chan, T.M.: Random sampling, halfspace range reporting, and construction of $(\leq k)$-levels in three dimensions. SIAM J. Comput. 30(2), 561-575 (2000)

8. Chan, T.M.: Three problems about simple polygons. Comput. Geom. Theory Appl. 35(3), 209-217 (2006)

9. Chazelle, B.: Filtering search: a new approach to query-answering. SIAM J. Comput. 15(3), 703-724 (1986)

10. Chazelle, B.: Triangulating a simple polygon in linear time. Discrete Comput. Geom. 6(5), 485-524 (1991)

11. Chazelle, B.: An optimal algorithm for intersecting three-dimensional convex polyhedra. SIAM J. Comput. 21(4), 671-696 (1992)

12. Chazelle, B.: The Discrepancy Method: Randomness and Complexity. Cambridge University Press, New York (2000)

13. Chazelle, B., Devillers, O., Hurtado, F., Mora, M., Sacristán, V., Teillaud, M.: Splitting a Delaunay triangulation in linear time. Algorithmica 34(1), 39-46 (2002)

14. Chew, L.P.: Building Voronoi diagrams for convex polygons in linear expected time. Tech. Rep. PCSTR90-147, Dartmouth College, Computer Science, Hanover, NH (1990)

15. Chew, L.P., Fortune, S.: Sorting helps for Voronoi diagrams. Algorithmica 18(2), 217-228 (1997)

16. Chin, F., Snoeyink, J., Wang, C.A.: Finding the medial axis of a simple polygon in linear time. Discrete Comput. Geom. 21(3), 405-420 (1999)

17. Chin, F., Wang, C.A.: Finding the constrained Delaunay triangulation and constrained Voronoi diagram of a simple polygon in linear time. SIAM J. Comput. 28(2), 471-486 (1998)

18. Clarkson, K.L.: A randomized algorithm for closest-point queries. SIAM J. Comput. 17(4), 830-847 (1988)

19. Clarkson, K.L., Shor, P.W.: Applications of random sampling in computational geometry. II. Discrete Comput. Geom. 4(5), 387-421 (1989)

20. Cormen, T.H., Leiserson, C.E., Rivest, R.L., Stein, C.: Introduction to Algorithms, 3rd edn. MIT Press, Cambridge (2009)

21. Devillers, O.: Randomization yields simple $O\left(n \log ^{*} n\right)$ algorithms for difficult $\Omega(n)$ problems. Int. J. Comput. Geom. Appl. 2(1), 97-111 (1992)

22. Djidjev, H.N., Lingas, A.: On computing Voronoi diagrams for sorted point sets. Int. J. Comput. Geom. Appl. 5(3), 327-337 (1995)

23. Dobkin, D.P., Kirkpatrick, D.G.: Fast detection of polyhedral intersection. Theor. Comput. Sci. 27(3), 241-253 (1983)

24. Dobkin, D.P., Kirkpatrick, D.G.: A linear algorithm for determining the separation of convex polyhedra. J. Algorithms 6(3), 381-392 (1985)

25. Fournier, H., Vigneron, A.: A tight lower bound for computing the diameter of a 3D convex polytope. Algorithmica 49(3), 245-257 (2007)

26. Kirkpatrick, D.G., Klawe, M.M., Tarjan, R.E.: Polygon triangulation in $O(n \log \log n)$ time with simple data structures. Discrete Comput. Geom. 7(4), 329-346 (1992)

27. Klein, R., Lingas, A.: A linear-time randomized algorithm for the bounded Voronoi diagram of a simple polygon. Int. J. Comput. Geom. Appl. 6(3), 263-278 (1996)

28. van Kreveld, M.J., Löffler, M., Mitchell, J.S.B.: Preprocessing imprecise points and splitting triangulations. In: Proc. 19th Annu. Internat. Sympos. Algorithms Comput. (ISAAC), pp. 544-555 (2008)

29. Matoušek, J.: Lectures on Discrete Geometry. Graduate Texts in Mathematics, vol. 212. Springer, New York (2002) 
30. Mulmuley, K.: Computational Geometry: An Introduction Through Randomized Algorithms. Englewood Cliffs, Prentice-Hall (1994)

31. Ramos, E.A.: On range reporting, ray shooting and $k$-level construction. In: Proc. 15th Annu. ACM Sympos. Comput. Geom. (SoCG), pp. 390-399 (1999)

32. Seidel, R.: A method for proving lower bounds for certain geometric problems. Tech. Rep. TR84-592, Cornell University, Ithaca, NY, USA (1984)

33. Seidel, R.: A simple and fast incremental randomized algorithm for computing trapezoidal decompositions and for triangulating polygons. Comput. Geom. Theory Appl. 1(1), 51-64 (1991) 